Revue québécoise de droit international

Quebec Journal of International Law

Revista quebequense de derecho internacional

\title{
Le régime des obligations positives de prévenir et de poursuivre à défaut d'extrader ou de remise prévues dans le texte des projets d'articles sur les crimes contre l'humanité provisoirement adoptés par la Commission du droit international
}

\section{Ezéchiel Amani Cirimwami et Stefaan Smis}

Volume 30, numéro 1, 2017

URI : https://id.erudit.org/iderudit/1053756ar

DOI : https://doi.org/10.7202/1053756ar

Aller au sommaire du numéro

Éditeur(s)

Société québécoise de droit international

ISSN

0828-9999 (imprimé)

2561-6994 (numérique)

Découvrir la revue

Citer cet article

Cirimwami, E. A. \& Smis, S. (2017). Le régime des obligations positives de prévenir et de poursuivre à défaut d'extrader ou de remise prévues dans le texte des projets d'articles sur les crimes contre l'humanité provisoirement adoptés par la Commission du droit international. Revue québécoise de droit international / Quebec Journal of International Law / Revista quebequense de derecho internacional, 30(1), 1-39. https://doi.org/10.7202/1053756ar
Résumé de l'article

Cet article concerne le texte des projets d'articles sur les crimes contre l'humanité provisoirement adopté par la Commission du droit international à sa soixante-huitième session en 2016. Il n'est pas exclu que le texte adopté connaisse ultérieurement des modifications tenant compte de l'évolution des discussions au sein de la Commission du droit international. La version finale du texte des projets d'articles est très attendu par les internationalistes d'autant plus qu'en 2014, la Commission faisait déjà observer dans ce qui constitue un « Rapport final » sur le thème de l'obligation d'extrader ou de poursuivre (aut dedere aut judicare) que le régime conventionnel existant présentait d'importantes lacunes quant à l'obligation d'extrader ou de poursuivre qu'il pourrait être nécessaire de combler. Elle relevait notamment l'absence de conventions internationales comportant cette obligation à l'égard de la plupart des crimes contre l'humanité. Aussi, rappellera-t-elle avoir inscrit ce sujet à son programme de travail, dans le cadre duquel il était envisagé d'élaborer un nouvel instrument portant sur la prévention et la répression des crimes contre l'humanité dont l'un des éléments serait une obligation d'extrader ou de poursuivre les auteurs de ces crimes. Le texte tel qu'adopté pose clairement, d'une part, que les crimes contre l'humanité, qu'ils soient ou non commis en temps de conflit armé, sont des crimes au regard du droit international que les États s'engagent à prévenir et à punir. D'autre part, que les États doivent coopérer à cette fin notamment au moyen de l'obligation de poursuivre ou d'extrader ou de remise. Toutefois, il appert de l'analyse du texte adopté qu'il n'existe à charge des États ni obligation de juger les présumés auteurs, ni celle de les punir. L’obligation consiste en réalité pour l'État à soumettre l'affaire à ses autorités compétentes pour l'exercice de l'action pénale. Ceci ne veut pas dire non plus une obligation d'engager des poursuites dans la mesure où ces autorités gardent sur l'affaire, la maîtrise du déclenchement des poursuites. Elles sont aussi libres de classer celle-ci sans suite sans que cette décision contrevienne à l'obligation imposée à l'État dont elles relèvent, de poursuivre le suspect à défaut de l'extrader ou de le remettre à une juridiction internationale compétente.
Ce document est protégé par la loi sur le droit d'auteur. L’utilisation des services d'Érudit (y compris la reproduction) est assujettie à sa politique d'utilisation que vous pouvez consulter en ligne.

https://apropos.erudit.org/fr/usagers/politique-dutilisation/ 


\title{
LE RÉGIME DES OBLIGATIONS POSITIVES DE PRÉVENIR ET DE POURSUIVRE À DÉFAUT D'EXTRADER OU DE REMISE PRÉVUES DANS LE TEXTE DES PROJETS D'ARTICLES SUR LES CRIMES CONTRE L'HUMANITÉ PROVISOIREMENT ADOPTÉS PAR LA COMMISSION DU DROIT INTERNATIONAL
}

\author{
Ezéchiel Amani Cirimwami* et Stefaan Smis ${ }^{* *}$
}

\begin{abstract}
Cet article concerne le texte des projets d'articles sur les crimes contre l'humanité provisoirement adopté par la Commission du droit international à sa soixante-huitième session en 2016. Il n'est pas exclu que le texte adopté connaisse ultérieurement des modifications tenant compte de l'évolution des discussions au sein de la Commission du droit international. La version finale du texte des projets d'articles est très attendu par les internationalistes d'autant plus qu'en 2014, la Commission faisait déjà observer dans ce qui constitue un «Rapport final» sur le thème de l'obligation d'extrader ou de poursuivre (aut dedere aut judicare) que le régime conventionnel existant présentait d'importantes lacunes quant à l'obligation d'extrader ou de poursuivre qu'il pourrait être nécessaire de combler. Elle relevait notamment l'absence de conventions internationales comportant cette obligation à l'égard de la plupart des crimes contre l'humanité. Aussi, rappellera-t-elle avoir inscrit ce sujet à son programme de travail, dans le cadre duquel il était envisagé d'élaborer un nouvel instrument portant sur la prévention et la répression des crimes contre l'humanité dont l'un des éléments serait une obligation d'extrader ou de poursuivre les auteurs de ces crimes. Le texte tel qu'adopté pose clairement, d'une part, que les crimes contre l'humanité, qu'ils soient ou non commis en temps de conflit armé, sont des crimes au regard du droit international que les États s'engagent à prévenir et à punir. D'autre part, que les États doivent coopérer à cette fin notamment au moyen de l'obligation de poursuivre ou d'extrader ou de remise. Toutefois, il appert de l'analyse du texte adopté qu'il n'existe à charge des États ni obligation de juger les présumés auteurs, ni celle de les punir. L'obligation consiste en réalité pour l'État à soumettre l'affaire à ses autorités compétentes pour l'exercice de l'action pénale. Ceci ne veut pas dire non plus une obligation d'engager des poursuites dans la mesure où ces autorités gardent sur l'affaire, la maîtrise du déclenchement des poursuites. Elles sont aussi libres de classer celle-ci sans suite sans que cette décision contrevienne à l'obligation imposée à l'État dont elles relèvent, de poursuivre le suspect à défaut de l'extrader ou de le remettre à une juridiction internationale compétente.
\end{abstract}

This article is about the text of the Draft Articles on Crimes Against Humanity temporarily adopted by the International Law Commission at its sixty-eighth session in 2016. It cannot be ruled out that the adopted text could subsequently be amended to take into account the evolution of the discussions within the International Law Commission. The final version of the Draft Articles ishighly anticipated by internationalists, all the more since in 2014, the Commission already observed in what constituted a "Final Report" on the theme of the obligation to extradite or prosecute (aut dedere aut judicare) that the existing treaty regime contained significant limitations as concerns the obligation to extradite or prosecute, which might need to be addressed. Amongst others, the Commission noted the absence of international treaties containing this obligation as relates to crimes against humanity. Also, the Commission reminded that it had placed this issue on its work programme, in the context of which was considered the elaboration of a new

* Doctorant en droit international à la Faculté de droit et de criminologie de la Vrije Universiteit Brussel. Ancien Substitut du Procureur de la République et actuellement, Juge en République Démocratique du Congo. E-mail : Ezechiel.Amani.Cirimwmami@vub.be.

** Professeur de droit international et de droits de l'homme à la faculté de droit et de criminologie de la Vrije Universiteit Brussel et Reader en droit international à la School of law de l'Université de Westminster. E-mail: Stefaan.Smis@vub.be.

Les auteurs remercient les lecteurs anonymes de la RQDI ainsi que Junior A Mumbala, Sa B Traoré et Trésor M Maheshe pour leurs commentaires sur la version antérieure du manuscrit. 
instrument on the prevention and repression of crimes against humanity that would include an obligation to extradite or prosecute the authors of these crimes. As adopted to this day, the text clearly provides, on the one hand, that crimes against humanity, should they be committed in times of armed conflict or not, are crimes under international law that states have committed to prevent and punish. On the other hand, it provides that states must cooperate in this respect, including through the obligation to prosecute or to extradite or to surrender. However, as appears from this analysis, there exists no obligation for states to try the presumed authors, nor to punish them. The obligation actually consists of the state submitting the case to its competent authorities for the exercise of criminal action. This does not mean, neither, that there exists an obligation to bring criminal charges, inasmuch as these authorities retain control on the initiation of proceedings. They are also free to not pursue the case without this decision resulting in a breach of the obligation imposed on the state under which they are acting, to prosecute the suspect without extraditing him or her, or to surrender him or her to a competent international jurisdiction.

Este artículo trata del proyecto de artículos sobre crímenes contra la humanidad temporalmente adoptado por la Comisión del derecho internacional en su $68^{\circ}$ período de sesiones en 2016 . No se puede excluir que el texto adoptado se enmiende de manera a tener en cuenta la evolución de las discusiones dentro de la Comisión del derecho internacional. La versión final de este texto es muy esperada por los internacionalistas ya que en 2014, la Comisión ya hacía observar en lo que constituye un " Informe final " sobre el tema de la obligación de extraditar o de perseguir (aut dedere aut judicare) que el régimen convencional existente presentaba lagunas importantes en cuanto a la obligación de extraditar o de perseguir que podría ser necesario colmar. Ella hacia ver particularmente la ausencia de convenios internacionales que contenía esta obligación con respecto a la inmensa mayoría de los crímenes contra la humanidad. También, recordará haber inscrito este sujeto en su programa de trabajo, en el marco del cual estaba previsto elaborar un nuevo instrumento que se refería en la prevención y la represión de los crímenes contra la humanidad de la que uno de los elementos sería una obligación de extraditar o de perseguir a los autores de estos crímenes. El texto tal como fue adoptado este día, afirma claramente, de una parte, que los crímenes contra la humanidad, sean o no cometidos en el tiempo de conflicto armado, son crímenes respecto al derecho internacional que los Estados se comprometen a prevenir y a castigar. Por otra parte, que los Estados deben cooperar a este fin particularmente por medio de la obligación de perseguir o de extraditar o de entregar. No obstante, aprece segun el análisis que no existe a cargo de los Estados obligación de juzgar ni los autores presuntos, ni de castigarlos. La obligación consiste en realidad para el Estado que somete el asunto a sus autoridades competentes al ejercicio de la acción penal. Esto no quiere decir tampoco una obligación de comprometer persecuciones en la medida en que estas autoridades guardan sobre el asunto, el control del inicio de las persecuciones. Son tan libres de clasificar éste sin continuación sin que esta decisión contravenga la obligación impuesta al Estado del que dependen, de perseguir al sospechoso a falta de extraditarlo o de devolverlo a una jurisdicción internacional competente. 
Dans le domaine du droit international, trois crimes particulièrement graves à savoir, le génocide, les crimes de guerre et les crimes contre l'humanité ont émergé et relèvent de la compétence des principaux tribunaux pénaux internationaux créés à ce jour ${ }^{1}$. Toutefois, seuls le génocide et les crimes de guerre font l'objet d'un traité de caractère universel faisant obligation aux États de prévenir et de réprimer les actes visés, ainsi que de coopérer entre eux à cette fin $^{2}$. Les crimes de guerre ont été codifiés par les dispositions des Conventions de Genève de $1949^{3}$ et du Protocole $I^{4}$ additionnel à ces instruments, relatives aux « infractions graves », et le génocide, par la Convention de 1948 sur la prévention et la répression crime de génocide. II n'existe aucun traité comparable en ce qui concerne les crimes contre l'humanité. Une convention de caractère universel fait nettement défaut ${ }^{6}$. Ceci explique qu'en 2014, la

1 Voir Rapport de la Commission du droit international sur les travaux de sa 65e session, Doc off AG NU, $68^{\mathrm{e}}$ sess, supp $\mathrm{n}^{\circ} 10$, Doc NU A/68/10 (2013) annexe B, à la p 154 au para 1 [Rapport de la CDI sur les travaux de sa $65^{e}$ session]. Un quatrième crime particulièrement grave s'inscrit à son tour dans le domaine de compétence de la Cour pénale internationale - le crime d'agression - mais le mécanisme d'exercice de la compétence de la Cour à son égard n'est pas à ce jour activé par les États. Il convient de signaler, toutefois, que le nombre exigé de 30 ratifications a été atteint le 26 juin 2016 à l'occasion du dépôt par l'État de Palestine de son instrument de ratification des amendements de Kampala au Statut de Rome en ce qui concerne le crime d'agression. Conformément à l'article 15 bis, point 3, inséré dans le Statut de Rome par la Résolution RC/Res.6, la Cour ne pourra exercer sa compétence à l'égard dudit crime que sur décision unique qui sera prise par la majorité des États parties que celle requise pour l'adoption d'un amendement au statut et ce, après le $1^{\text {er }}$ janvier 2017 . Il y a lieu d'espérer que cette décision unique soit adoptée à la $16^{\mathrm{e}}$ Assemblée des Etats parties prévue à New-York en décembre 2017.

2 Voir également Leila Nadya Sadat, A Comprehensive History of the Proposed International Convention on the Prevention and Punishment of Crimes Against Humanity, Washington, Washington University Law, 2010 aux pp 3-4; Darryl Robinson, « The Draft Convention on Crimes Against Humanity: What to Do with the Definition? » dans Morten Bergsmo et Song Tianying, dir, On the Proposed Crimes Against Humanity Convention, Bruxelles, Torkel Opsahl Academic EPublisher, 2014 à la p 104 [Robinson]: "The Draft Convention is a welcome initiative for many reasons. War crimes and genocide are subjects of treaty obligations, whereas the third core crime-crimes against humanity-lacks the same clarity of enforcement obligations. This is particularly regrettable given that crimes against humanity are of the greatest contemporary relevance (as they do not require armed conflict or special genocidal intent). A convention would remove ambiguities about the obligation to prosecute and about jurisdictional rules ».

3 Convention I de Genève pour l'amélioration du sort des blessés et des malades dans les forces armées en campagne, 12 août 1949, 75 RTNU 31 (entrée en vigueur : 21 octobre 1950), art 50; Convention II de Genève pour l'amélioration du sort des blessés, des malades et des naufragés des forces armées sur mer, 12 août 1949, 75 RTNU 85 (entrée en vigueur : 21 octobre 1950), art 51; Convention III de Genève relative au traitement des prisonniers de guerre, 12 août 1949, 75 RTNU 135 (entrée en vigueur : 21 octobre 1950), art 130; Convention IV de Genève relative à la protection des personnes civiles en temps de guerre, 12 août 1949, 75 RTNU 287 (entrée en vigueur : 21 octobre 1950), art 147 [Conventions de Genève].

$4 \quad$ Protocole additionnel I aux Conventions de Genève du 12 août 1949 relatif à la protection des victimes des conflits armés internationaux, 8 juin 1977, 1125 RTNU 3 (entrée en vigueur : 7 décembre 1978), arts 11 et 85 [Protocole additionnel 1 ].

5 Convention pour la prévention et la répression du crime de génocide, 9 décembre 1948, 78 RTNU 277 (entrée en vigueur : 12 janvier 1951), art I [Convention sur le génocide].

6 Cherif M Bassiouni, "Revisiting the Architecture of Crimes Against Humanity: Almost a Century in the Making, with Gaps and Ambiguities Remaining - the Need for a Specialized Convention » dans Leila Nadya Sadat, dir, Forging a Convention for Crimes Against Humanity, Cambridge, Cambridge University Press, 2011 à la p 58 : " It is nothing short of amazing that since World War II, crimes against humanity has not been codified in an international convention ». 
Commission de droit international (CDI) a fait observer, dans son rapport final sur le thème de l'obligation d'extrader ou de poursuivre (aut dedere aut judicare) ${ }^{7}$, que

le régime conventionnel existant présente d'importantes lacunes quant à l'obligation d'extrader ou de poursuivre qu'il pourrait être nécessaire de combler, [relevant] notamment l'absence de conventions internationales comportant cette obligation à l'égard de la plupart des crimes contre l'humanité8.

Aussi, rappellera-t-elle avoir inscrit ce sujet à son programme de travail ${ }^{9}$, « dans le cadre duquel il est envisagé d'élaborer un nouvel instrument [portant sur la prévention et la répression des crimes contre l'humanité] dont l'un des éléments serait une obligation d'extrader ou de poursuivre les auteurs de crimes de cette nature ${ }^{10} » . \grave{A}$ la suite de ses travaux sur la question ${ }^{11}$, la CDI a provisoirement adopté tant le texte

7 Rapport final de la CDI sur le sujet " obligation d'extrader ou de poursuivre (aut dedere aut judicare) ", Doc off AG NU, 69 ${ }^{\mathrm{e}}$ sess, supp n¹0, Doc NU A/69/10 (2014) aux pp 144-70 [Rapport final de la CDI-aut dedere aut judicare].

8 Ibid à la p 153 au para 14. Pour une lecture plus critique dudit « Rapport final», voir Lucie Delabie, «Les conclusions de la Commission du droit international au sujet de l'obligation d'extrader ou de poursuivre (aut dedere aut judicare) : un 'Rapport final' aux finalités obscures » (2015) 15 AFDI 375 [Delabie].

9 À sa $65^{\mathrm{e}}$ session, en 2013, la CDI a décidé d'inscrire le sujet «Crimes contre l'humanité » à son programme de travail à long terme (voir Rapport de la CDI sur les travaux de sa $65^{e}$ session, supra note 1 à la p 120 au para 170 et annexe B). À l'issue d'un débat au sein de la Sixième Commission en 2013 (voir le Rapport de la CDI sur les travaux de ses $63^{e}$ et $65^{e}$ sessions : Résumé thématique, établi par le Secrétariat, des débats tenus par la Sixième Commission de l'Assemblée générale à sa $68^{\mathrm{e}}$, A/CN.4/666 [2014] à la p 19 au para 72), l'Assemblée générale a pris note de cette décision (voir Rés AG 68/112, Doc off NU, 68 ${ }^{\mathrm{e}}$ sess, Doc NU A/RES/68/112 [2013] au para 8). À sa $66^{\mathrm{e}}$ session (2014), la Commission a décidé d'inscrire le sujet «Crimes contre l'humanité » à son programme de travail en cours et a désigné M Sean D. Murphy Rapporteur spécial pour le sujet (voir, Rapport final de la CDIaut dedere aut judicare, supra note 7 au para 266). À l'issue d'un débat au sein de la Sixième Commission en 2014, l'Assemblée générale a pris note de cette nouvelle décision (voir Ibid à la p 276 au para 266; et Rés AG 69/118, Doc off NU, 69e sess, Doc NU A/RES/69/118 [2014] au para 7).

10 Rapport final de la CDI-aut dedere aut judicare, supra note 7 à la p 160 au para 31 .

11 À sa $67^{\mathrm{e}}$ session (2015), la Commission a examiné le premier rapport du Rapporteur spécial (Sean D Murphy, Rapporteur spécial, Premier rapport sur les crimes contre l'humanité, Doc off AG NU, $67^{\mathrm{e}}$ sess, Doc NU A/CN.4/680 [Murphy, Premier rapport sur les crimes contre l'humanité]) et a provisoirement adopté quatre projets d'article et les commentaires y relatifs (voir Texte des projets d'articles sur les crimes contre l'humanité provisoirement adoptés à ce jour par la Commission, Doc off AG NU, 70 ${ }^{\mathrm{e}}$ sess, supp n¹0, Doc NU A/70/10 [2015] aux paras 110-17 [Projets d'articles sur les crimes contre l'humanité]). Elle a aussi demandé au Secrétariat d'établir un mémoire contenant des informations sur les mécanismes conventionnels de suivi qui pourraient être pertinents pour ses travaux futurs sur le sujet (Ibid, au para 115). À sa $68^{\mathrm{e}}$ session (2016), la Commission était saisie du deuxième rapport du Rapporteur spécial (A/CN.4/690), ainsi que du mémoire du Secrétariat présentant des informations sur les mécanismes conventionnels de suivi qui pourraient être pertinents pour les travaux futurs de la CDI (A/CN.4/698), qu'elle a examinés à ses $3296^{\mathrm{e}}$ à $3301^{\mathrm{e}}$ séances, du 11 au 19 mai 2016 (voir les comptes rendus analytiques provisoires des $3296^{\mathrm{e}}$ à $3301^{\mathrm{e}}$ séances : A/CN.4/SR.3296, A/CN.4/SR.3297, A/CN.4/SR.3298, A/CN.4/SR.3299, A/CN.4/SR.3300 et A/CN.4/SR.3301). Dans son deuxième rapport, le Rapporteur spécial traitait de l'incrimination en droit interne (chap I), de l'établissement de compétence interne (chap II), de l'enquête générale et de la coopération aux fins de l'identification de l'auteur présumé (chap III), de l'exercice par l'État de sa compétence nationale lorsque l'auteur présumé se trouve sur son territoire (chap IV), du principe aut dedere aut judicare (chap V), du traitement équitable de l'auteur présumé de l'infraction (chap VI) et du programme de travail futur sur le sujet (chap VII). Le Rapporteur spécial proposait six projets d'article correspondant 
des projets d'articles sur les crimes contre l'humanité ${ }^{12}$ que les commentaires y relatifs ${ }^{13}$. Bien que provisoires, ces projets d'articles sont appelés à devenir une convention sur la prévention et la répression des crimes contre l'humanité ${ }^{14}$. Dès lors, l'intérêt de cette étude est évident au vu de l'actualité de ce projet de Convention et étant entendu que les États ont clairement affirmé que les travaux sur ce sujet contribueraient au développement du droit pénal international ${ }^{15}$ et compléteraient les travaux antérieurs de la $\mathrm{CDI}^{16}$, notamment en ce qui concerne la manière dont l'obligation d'extrader ou de poursuivre pourrait s'appliquer aux crimes contre l'humanité $^{17}$. Plusieurs commentateurs ${ }^{18}$ et organisations non gouvernementales ${ }^{19}$ se sont aussi intéressés aux différents aspects que le projet de convention devrait nécessairement couvrir, dont l'obligation aut dedere aut judicare ${ }^{20}$. Afin de

aux questions traitées dans les chapitres I à VI, respectivement [voir le deuxième rapport sur les crimes contre l'humanité (A/CN.4/690) : projet d'article 5 (Incrimination en droit interne); projet d'article 6 (Établissement de la compétence nationale); projet d'article 7 (Enquête générale et coopération aux fins de l'identification de l'auteur présumé); projet d'article 8 (Exercice par l'État de sa compétence nationale lorsque l'auteur présumé de l'infraction se trouve sur son territoire); projet d'article 9 (Principe aut dedere aut judicare); et projet d'article 10 (Traitement équitable de l'auteur présumé de l'infraction)]. À sa $3301^{\mathrm{e}}$ séance, le 19 mai 2016, la Commission a renvoyé au Comité de rédaction les projets d'articles 5, 6, 7, 8, 9 et 10 figurant dans le deuxième rapport du Rapporteur spécial. Il a aussi prié le Comité de rédaction d'examiner la question de la responsabilité pénale des personnes morales sur la base d'un document de réflexion à élaborer par le Rapporteur spécial. À ses $3312^{\mathrm{e}}$ et $3325^{\mathrm{e}}$ séances, les 9 juin et 21 juillet 2016, respectivement, la Commission a examiné deux rapports du Comité de rédaction et a provisoirement adopté les projets d'articles 5 à 10 . À sa $3341^{\mathrm{e}}$ séance, le 9 août 2016, la Commission a adopté les commentaires des projets d'article provisoirement adoptés.

12 Projets d'articles sur les crimes contre l'humanité, supra note 11; Doc off AG NU, $71^{\mathrm{e}}$ sess, supp $\mathrm{n}^{\circ} 10$, Doc NU A/71/10 (2016) aux pp 253-91.

13 Ibid.

14 Le premier article, «Champ d'application », serait défini comme suit : « Les présents projets d'article s'appliquent à la prévention et à la répression des crimes contre l'humanité » [Nos italiques]. L'objectif de la CDI est de présenter une proposition définitive de convention sur les crimes contre l'humanité à l'horizon 2019. En ce sens, voir Rébecca Mignot-Mahdavi, « Vers une convention sur les crimes contre l'humanité » dans Julián Fernández, dir, Justice pénale internationale, Paris, CNRS Editions, 2016 à la p 97 [Mignot-Mahdavi].

15 Voir notamment, Croatie (A/C.6/69/SR.20 au para 94); Japon (Ibid au para 49).

16 Croatie (Ibid aux paras 94-97); République tchèque (Ibid au para 10).

17 Chili (A/C.6/69/SR.24 au para 52); Finlande (au nom des pays nordiques) (A/C.6/69/SR.19 au para 82); Royaume-Uni (Ibid au para 159).

18 Voir notamment, Leila Nadya Sadat, dir, Forging a Convention for Crimes Against Humanity, Cambridge, Cambridge University Press, 2011 [Sadat]; Morten Bergsmo et Song Tianying, dir, On the Proposed Crimes Against Humanity Convention, Bruxelles, Torkel Opsahl Academic EPublisher, 2014; Mignot-Mahdavi, supra note 14 aux pp 97-111.

19 Letter to Professor Sean Murphy, Special Rapporteur of the International Law Commission on Crimes Against Humanity from Leading NGOs, 16 February 2016, cité dans Ibid à la p 97 : «We believe that provisions on aut dedere aut judicare and universal jurisdiction are essential aspects of the proposed Convention and it is vital that the Convention does not backtrack on such important international law principles ».

20 Ian Kenned, «The Proposed Convention on Crimes Against Humanity and Aut Dedere Aut Judicare » dans Morten Bergsmo et Song Tianying, dir, On the Proposed Crimes Against Humanity Convention, Bruxelles, Torkel Opsahl Academic EPublisher, 2014, 329 [Kenned]; Shang Weiwei and Zhang Yueyao, «The Aut Dedere Aut Judicare Provision in the Proposed Convention on Crimes Against Humanity: Assessment from a Chinese perspective » dans Morten Bergsmo et Song Tianying, dir, On the Proposed Crimes Against Humanity Convention, Bruxelles, Torkel Opsahl Academic EPublisher, 2014 aux pp 345-66. 
comprendre le nouveau développement apporté au droit international quant au régime de l'obligation d'extrader ou de poursuivre les crimes contre l'humanité, il importe d'examiner sous l'angle de ce nouveau cadre, à savoir le texte des projets d'articles provisoirement adoptés par la CDI, censé répondre aux insuffisances décriées, le contenu, et le sens de l'obligation aut dedere aut judicare qui en découle. Mais pour y arriver, il semble approprié d'examiner comment ce «nouveau » cadre répressif définit les crimes contre l'humanité (I) avant d'envisager faire l'état de l'obligation de prévenir ces crimes (II) et de s'attarder sur l'obligation de les poursuivre (III) dans le but de dégager clairement le contenu, le sens de l'obligation et sa mise en œuvre.

\section{Un nouveau cadre répressif et une redéfinition des crimes contre l'humanité dans les projets d'article provisoirement adopté par la Commission du droit international ?}

$\mathrm{Si}$ aucune convention internationale à caractère normatif n'existe sur la prévention et la répression des crimes contre l'humanité, la notion elle-même de « crimes contre l'humanité » ne paraît pas nouvelle. Elle plonge ses racines loin dans le temps lorsque l'on pense à la «clause de Martens » figurant dans la Convention concernant les lois et coutumes de la guerre sur terre et dans la Convention (IV) concernant les lois et coutumes de la guerre sur terre (Conventions de La Haye de 1899 et 1907). Cette dernière, s'agissant de protéger les personnes en temps de guerre, faisait référence aux « lois de l'humanité et [aux] exigences de la conscience publique $^{21} \gg$. On entend généralement par cette disposition que tant que les lois de la guerre ne sont pas entièrement codifiées, les principes d' "humanité » offrent une protection résiduelle ${ }^{22}$. La notion a évolué au fil des années avec les instruments historiques, à caractère procédural, qui en constituent le cadre répressif. Ce sont notamment les statuts des tribunaux militaires internationaux de Nuremberg (Statut du Tribunal de Nuremberg) et pour l'Extrême-Orient (Statut du Tribunal de Tokyo), en passant par les statuts de deux tribunaux pénaux internationaux ad hoc pour l'exYougoslavie (TPIY) et le Rwanda (TPIR) jusqu'au statut de la Cour pénale internationale (Statut de Rome $)^{23}$. Aussi, a-t-elle reçu une attention particulière au sein

21 Convention (IV) concernant les lois et coutumes de la guerre sur terre, 18 octobre 1907, préambule, Actes et documents de la Deuxième Conférence internationale de la paix (La Haye, 15 juin18 octobre 1907), La Haye, Imprimerie nationale, 1907, t I. Dans sa version de 1907, la clause se lit comme suit : «En attendant qu'un code plus complet des lois de la guerre puisse être édicté, les Hautes Parties contractantes jugent opportun de constater que, dans les cas non compris par les dispositions réglementaires adoptées par elles, les populations et les belligérants restent sous la sauvegarde et sous l'empire des principes du droit des gens, tels qu'ils résultent des usages établis entre nations civilisées, des lois de l'humanité et des exigences de la conscience publique ».

22 Ibid. Voir Theodor Meron, «The Martens Clause, Principles of Humanity, and Dictates of Public Conscience » (2000) 94:1 AJIL 78; Rupert Ticehurst, « La clause de Martens et le droit des conflits armés » (1997) 79:824 RICR 133.

23 Pour une discussion plus détaillée de l'historique du crime contre l'humanité, voir Murphy, Premier rapport sur les crimes contre l'humanité, supra note 11 aux paras 15-51. 
de la doctrine ${ }^{24}$, avant que le sujet ayant conduit au présent texte de projets d'article provisoirement adopté ne soit inscrit à l'ordre du jour de la $\mathrm{CDI}^{25}$. Aux termes du Statut de Rome, les crimes contre l'humanité ont été définis comme l'un quelconque des actes énumérés à l'article 7 lorsqu'il est commis dans le cadre d'une attaque généralisée ou systématique lancée contre toute population civile et en connaissance de cette attaque ${ }^{26}$.

Cette définition a toutefois fait l'objet de vives critiques tant et si bien que, quelle qu'en soit la lecture, elle n'en finit pas avec les controverses auxquelles il était attendu que les présents projets d'articles répondent ${ }^{27}$. L'on peut relever, en l'occurrence, les divergences autour de l'élément politique ${ }^{28}$ dérivant de l'article 7(2) du Statut de Rome. Cet élément impose que pour être qualifiée de crime contre l'humanité, une attaque doit être liée à une politique d'État ou d'organisation. Ceci a toujours divisé la doctrine lorsque les commentateurs cherchent à savoir si le terme «politique d'une organisation » signifie que les organisations non étatiques peuvent commettre des crimes contre l'humanité. Si Cherif M Bassiouni est ferme à ce sujet en soutenant que :

The crimes against humanity definition clearly refers to State policy, and the words 'organizational policy' do not refer to the policy of an organization, but the policy of a State. It does not refer to non-State actors $^{29}$.

24 Voir notamment Cherif M Bassiouni, Crimes Against Humanity: Historical Evolution and Contemporary Application, Cambridge, Cambridge University Press, 2011; Norman Geras, Crimes Against Humanity: Birth of a Concept, Manchester, New York, Manchester University Press, 2011; Philippe Currat, Les crimes contre l'humanité dans le Statut de la Cour pénale internationale, Bruxelles, Bruylant, 2006; Antonio Cassese, "Crimes Against Humanity » dans Antonio Cassese et al, dir, The Rome Statute of the International Criminal Court: A Commentary, Oxford, Oxford University Press, 2002 à la p 375 [Cassese, «Crimes Against Humanity»]; Mario Bettati, « Le crime contre l'humanité » dans Hervé Ascension, Emmanuel Decaux et Allain Pellet, dir, Droit international pénal, Paris, Pedone, 2000 aux pp 293-371; Darryl Robinson, «Defining Crimes Against Humanity at the Rome Conference» (1999) 93:1 AJIL 43; Elisabeth Zoller, «La définition des crimes contre l'humanité » (1993) 120:3-4 JDI 349; Sadat, supra note 18.

25 Voir Rapport de la CDI sur les travaux de sa $65^{e}$ session, supra note 1; Rapport final de la CDI-aut dedere aut judicare, supra note 7.

26 Statut de Rome de la Cour pénale internationale, 17 juillet 1998, 2187 RTNU 3 (entrée en vigueur : $1^{\text {er }}$ juillet 2002), art 7(1) [Statut de Rome]

27 Voir Robinson, supra note 2 à la p 103: «The Article 7 definition features the now-iconic contextual element of a 'widespread or systematic attack directed against a civilian population'. The definition of 'attack', in Article 7(2)(a), requires a 'State or organizational policy'. This raises legitimate questions about the relationship between 'policy' and 'systematic', whether they are duplicative, and whether the policy element will complicate and restrict crimes against humanity prosecutions. This chapter will look both at the logical structure of Article 7 and at past authorities to show that 'policy' is, and must be, different from and less demanding than 'systematic' ".

28 À ce propos, voir Christopher Roberts, «The Definition of Crimes Against Humanity and Other Widespread or Systematic Human Rights Violations » (2017) 20:1 University of Pennsylvania Journal of Law and Social Change 1; Yves Hamuli Kabumba, «L'élément politique des crimes contre l'humanité : État des lieux de la jurisprudence de la Cour pénale internationale » (2015) 52:1 AFDI 223; Thomas Obel Hansen, « The Policy Requirement in Crimes Against Humanity: Lessons from and for the Case of Kenya », (2011) 43:1 Geo Wash Int'l L Rev 1.

29 Cherif M Bassiouni, The Legislative History of the International Criminal Court: Introduction, Analysis, and Integrated Text, Ardsley, Transnational Publishers, 2005 aux pp 151-52. 
Kai Ambos, par contre, adopte une interprétation contraire, et peut-être plus intuitive, de la même disposition :

[T] he reference to ["organizational policy"] in Article 7(2) [of the ICC Statute] makes clear that the provision also applies to non-State actors ${ }^{30}$.

Si Ambos soutient que la «politique d'une organisation » s'applique aussi aux acteurs non étatiques, il souligne toutefois que ces derniers doivent être en mesure d'agir comme un État, c'est-à-dire qu'ils doivent disposer des capacités d'organisation et de forces similaires à celles d'un État ${ }^{31}$. Comme le relève à juste titre Ian Kenned, ce débat laisse une question importante sans réponse : celles de savoir si les acteurs non étatiques peuvent commettre des crimes contre l'humanité et, dans l'affirmative, quelle structure et quelles capacités leurs organisations doivent-elles avoir ${ }^{32}$.

Par ailleurs, d'autres commentateurs ont soulevé la même préoccupation, mais cette fois en lien avec d'autres éléments de la définition, à l'instar de Madeleine Schwarz qui faisait observer, à ce propos, que

l'on ne sait pas exactement comment une définition exigeant que l'attaque consiste en la commission multiple d'actes et soit menée en application ou dans la poursuite d'une politique n'annule pas, dans une certaine mesure, tout bénéfice que pourrait procurer le fait de disposer d'un seuil en indiquant que l'attaque doit être soit généralisée soit systématique ${ }^{33}$.

D'autres questionnements relatifs notamment à la portée de la phrase « contre toute population civile » ont été soulevés quant à savoir, d'abord, dans quelle mesure un groupe de civils doit-il être qualifié de " population », ensuite, si on devait en même temps entendre par «civil», les personnes qui ne combattent plus ${ }^{34}$. Ce faisant, Darryl Robinson considérait que si les travaux de la CDI concernant la Convention sur les crimes contre l'humanité devaient s'inspirer de l'article 7 du Statut de Rome relativement à la définition des dits crimes, cette dernière pourrait être réécrite ou, à tout le moins, des commentaires importants pourraient y être consacrés afin d'en

30 Kai Ambos, «Crimes Against Humanity and the International Criminal Court » dans Leila Nadya Sadat, dir, Forging a Convention for Crimes Against Humanity, Cambridge, Cambridge University Press, 2011 à la $\mathrm{p} 281$ [Ambos].

31 Ibid à la p 283 («These actors must be in a position to act like a State, that is, they must possess a similar capacity of organization and force »).

32 Kenned, supra note 20 à la p 377. Voir également, Gerhard Werle et Boris Burghardt, « Do Crimes Against Humanity Require the Participation of a State or a 'State-like' Organisation » (2012) 10:1 Journal of International Criminal Justice 1151; Cherif M Bassiouni, « Revisiting the Architecture of Crimes Against Humanity: Almost a Century in the Making, with Gaps and Ambiguities Remaining the Need for a Specialized Convention » dans Leila Nadya Sadat, dir, Forging a Convention for Crimes Against Humanity, Cambridge, Cambridge University Press, 2011 à la p 5 [Bassiouni, «Revisiting the Architecture »]; Beth Van Schaack, « The Definition of Crimes Against Humanity: Resolving the Incoherence » (1999) 37:3 Colum J Transnat'1 L 787.

33 Madeleine Schwarz, "Prosecuting Crimes Against Humanity in Canada: What Must Be Proved» (2002) 46:1 Crim LQ 40 à la p 65 («It is unclear how a definition that requires that the attack involve the multiple commission of acts and be pursuant to or in furtherance of a policy does not to some degree cancel out any benefit of having a threshold that indicates that the attack must be either widespread or systematic »). Dans le même ordre d'idée, voir Ambos, supra note 30 aux pp 284-85.

34 Bassiouni, « Revisiting the Architecture », supra note 32. 


\section{élaguer les ambiguïtés ou les dangers liés à la mauvaise interprétation ${ }^{35}$.}

On peut effectivement constater que dans le cadre du texte des projets d'articles sous examen, la définition des crimes contre l'humanité s'est largement inspirée de l'article $7 \mathrm{du}$ Statut de Rome ${ }^{36}$. La volonté des États était clairement annoncée de ne pas s'en écarter ${ }^{37}$ en raison du grand nombre d'États qui y sont parties $^{38}$. Nonobstant le fait que les auteurs estiment parfois que l'article 7 du Statut de Rome pourrait être amélioré et malgré la divergence des avis sur le point de savoir s'il reflète ou non le droit international coutumier $^{39}$ ou quant à la meilleure

35 Robinson, supra note 2 à la p 106 («There are at least four advantages to this approach. First, commentary can facilitate acceptance by those who are concerned about the dangers of misinterpretation or over-extension of the policy element. Second, the commentary can facilitate prosecution and make the convention more effective, by demonstrating how the policy element has been understood and applied. Third, by drawing on national and international authorities, many of which are not well known, the commentary can help show that there is considerable harmony in the different authorities, and thus reduce the current fragmentation in the law. Fourth, as International Law Commission ('ILC') commentary is often used to aid in interpretation and as a guide to customary law, it will be of assistance not only in relation to the convention but also for national and international courts applying crimes against humanity law for any reason »).

36 Voir le commentaire 1) du projet d'article 3 : «Les trois premiers paragraphes de l'article 3 du projet établissent, aux fins du présent projet d'articles, une définition du «crime contre l'humanité ». Leur libellé est repris textuellement de celui de l'article 7 du Statut de Rome ». Projets d'articles sur les crimes contre l'humanité, supra note 11 à la p 61.

37 Tous les États qui se sont prononcés sur cette question devant la Sixième Commission à l'automne 2014 ont soutenu qu'il ne fallait pas introduire dans la nouvelle convention une définition de « crime contre l'humanité » qui s'écarte de celle retenue à l'article 7 du Statut de Rome. Voir Autriche (A/C.6/69/SR.19 au para 111); Croatie (A/C.6/69/SR.20 au para 94); Finlande (au nom des pays nordiques) (A/C.6/69/SR.19 au para 81); Italie (A/C.6/69/SR.22 au para 53); Pologne (A/C.6/69/SR.20 au para 36); Nouvelle-Zélande (A/C.6/69/SR.21 au para 33); République de Corée (Ibid au para 45) et Mongolie (A/C.6/69/SR.24 au para 94). Dans le même sens, Murphy, Premier rapport sur les crimes contre l'humanité, supra note 11 aux pp 6 au para 8, 10 au para 21 : "Considérant que la définition donnée à l'article 7 du Statut de Rome est désormais largement admise par les États, et par souci de favoriser l'harmonie des actions menées aux niveaux national et international pour lutter contre ces crimes, le projet d'article proposé reprend à l'identique la définition des " crimes contre l'humanité » figurant à l'article 7 [...] Il conviendrait en particulier d'éviter tout conflit entre cette convention et le Statut de Rome. Les travaux d'élaboration d'une nouvelle convention s'inspireront sûrement, le cas échéant, des dispositions du Statut de Rome et des instruments ainsi que de la jurisprudence qui y sont associés, mais il faut veiller à ce que les dispositions du nouvel instrument ne soient pas en contradiction avec le Statut de Rome, en raison du grand nombre d'États qui y sont parties ».

38 Le commentaire 8) de la CDI adopté en même temps que le projet d'article 3 auquel il réfère indique que la définition du « crime contre l'humanité » énoncée à l'article 7 du Statut de Rome a été acceptée par les plus de 120 États qui sont parties à ce statut, et bon nombre d'États la reprennent à présent lorsqu'ils adoptent ou modifient leurs lois nationales. Cette adoption est de 2015 (Projets d'articles sur les crimes contre l'humanité, supra note 11 à la p 63). Il convient de préciser qu'à ce jour, 124 États ont ratifié le Statut de Rome, la dernière ratification étant celle du El Salvador, intervenue le 3 Mars 2016. Toutefois, il convient aussi de noter que le Burundi, la RSA et la Gambie ont annoncé avoir amorcé le processus de retrait du Statut de Rome. Alors que ce processus était en cours et que le retrait conforme à l'article $127 \mathrm{du}$ Statut de Rome n'était pas encore effectif pour tous les trois États, la Gambie et la RSA ont respectivement notifié au Secrétaire Général de l'ONU, le 10 février et le 7 mars 2017, l'annulation de leur retrait de l'instrument fondateur de la CPI.

39 Voir par exemple Cassese, « Crimes Against Humanity », supra note 24. Bien plus, si l'article 7 du Statut de Rome fait référence à l'existence d'une «politique », la Chambre d'appel du Tribunal pénal international pour l'ex-Yougoslavie a jugé en 2002 dans l'affaire Kunarac que « rien » dans le droit international coutumier n'exigeait l'existence d'une politique et que le contraire en 
interprétation à donner à certains de ses aspects ${ }^{40}$, il n'a dès lors fait guère de doute au sein de la CDI que la définition de crime contre l'humanité qui y est énoncée est très largement admise par les États. Au demeurant, tous les États qui se sont prononcés sur cette question devant la Sixième Commission à l'automne 2014 ont soutenu qu'il ne fallait pas introduire dans la nouvelle convention une définition de « crime contre l'humanité » qui s'écarte de celle retenue à l'article 7 du Statut de Rome ${ }^{41}$. À l'appui de ce qui précède, le rapporteur spécial Sean Murphy relevait déjà dans son premier rapport que toute convention tendant en partie à promouvoir le régime de complémentarité du Statut de Rome devrait reprendre la définition de l'article 7 en vue de favoriser l'harmonisation des lois nationales avec le Statut. Plus généralement, l'utilisation de la définition de l'article 7 permettrait de minimiser le risque de fragmentation du droit pénal international ${ }^{42}$.

Ainsi dit, le projet d'article 3 consacré à la définition des crimes contre l'humanité a tout simplement repris, avec trois modifications de forme rendues nécessaires par la différence de contexte d'utilisation de la définition ${ }^{43}$, les dispositions de l'article 7 du Statut de Rome qui donne une définition des crimes contre l'humanité largement acceptée relevant du droit international établi ${ }^{44}$. Dès lors, il est impossible d'affirmer que les crimes contre l'humanité ont été redéfinis dans le cadre de ces projets d'articles. Ceux-ci restent, en effet, l'un quelconque des actes repris au point 1 de l'article $3^{45}$, et correspondant à l'article 7 du Statut de Rome, lorsqu'il est commis dans le cadre d'une attaque généralisée ou systématique lancée

ressortait même très «nettement» (Arrêt Kunarac au para 98). Néanmoins, avec le temps et l'adhésion d'un grand nombre d'États au Statut de Rome, il paraît vraisemblable que l'article 7 ait pour effet de cristalliser le droit international coutumier. Voir Richard Baxter, « Multilateral Treaties as Evidence of Customary International Law » (1965) 41 Brit YB Int'l L à la p 275.

40 Pour une vue globale, Robinson, supra note 2 aux pp 103, 105.

41 Voir notamment Autriche (A/C.6/69/SR.19 au para 111); Croatie (A/C.6/69/SR.20 au para 94); Finlande (au nom des pays nordiques) (A/C.6/69/SR.19 au para 81); Italie (A/C.6/69/SR.22 au para 53); Pologne (A/C.6/69/SR.20 au para 36); Nouvelle-Zélande (A/C.6/69/SR.21 par. 33); République de Corée (Ibid au para 45) et Mongolie (A/C.6/69/SR.24 au para 94).

42 Murphy, Premier rapport sur les crimes contre l'humanité, supra note 11 au para 122.

43 Voir à cet effet, le commentaire 8) du projet d'article 3 : «De fait, le texte de l'article 7 est repris textuellement à l'exception de trois modifications ne portant pas sur le fond, qui sont nécessaires compte tenu du contexte différent dans lequel la définition est employée. Tout d'abord, le début du paragraphe 1 est "Aux fins du présent projet d'articles" au lieu de "Aux fins du présent Statut". Deuxièmement, la même modification a été apportée au début du paragraphe 3 . Troisièmement, le paragraphe $1 \mathrm{~h}$ ) de l'article $7 \mathrm{du}$ Statut de Rome incrimine les actes de persécution lorsqu'ils sont commis "en corrélation avec tout acte visé dans le présent paragraphe ou tout crime relevant de la compétence de la Cour". Là aussi, pour tenir compte de la différence de contexte, ce membre de phrase est devenu, au projet d'article 3, "en corrélation avec tout acte visé dans le présent paragraphe ou du crime de génocide ou des crimes de guerre". Le moment venu, la CPI pourra exercer sa compétence sur le crime d'agression, une fois que les conditions fixées par la Conférence de Kampala seront remplies, auquel cas il faudra peut-être remanier le paragraphe ». Projets d'articles sur les crimes contre l'humanité, supra note 11 aux pp 63-64.

44 Voir par exemple, le Rapport de la Commission d'enquête du Conseil des droits de l'homme sur les

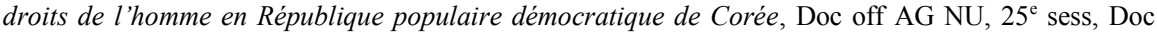
NU A/HRC/25/63 (2014) au para 21 : «Les questions touchant aux crimes contre l'humanité ont été étudiées sur la base des définitions issues du droit pénal international coutumier et énoncées dans le Statut de Rome de la Cour pénale internationale ».

45 Voir Projets d'articles sur les crimes contre l'humanité, supra note 11, art 3. 
contre toute population civile et en connaissance de cette attaque. À ce propos, il convient de préciser que la Commission a jugé prudent d'insérer le paragraphe 4 au projet d'article 3 qui dispose que ce dernier est sans préjudice de toute définition plus large prévue par tout instrument international ou loi nationale. En conséquence, si un État souhaite adopter une définition plus large dans son droit interne, le présent projet d'articles ne l'empêche pas de le faire ${ }^{46}$.

Néanmoins, la CDI semble avoir pris la mesure du problème suscité par les lectures controversées de certains aspects de la définition du crime contre l'humanité. On s'en aperçoit au regard des 41 commentaires qu'elle a réservés au seul projet d'article 3 et qui, à cet effet, font de lui, l'un des articles les plus commentés de l'ensemble du texte adopté ${ }^{47}$. Sans qu'il soit besoin de revenir sur l'ensemble de ces commentaires, l'on peut, relativement aux controverses précédemment rappelées, relever que la CDI, après avoir analysé - avec un soin minutieux de bénédictin - les décisions des juridictions pénales internationales, et rappelé ses travaux antérieurs sur des projets de codes, elle en conclut notamment :

En conséquence de la «politique» émanant éventuellement d'une organisation non étatique, la définition énoncée aux paragraphes 1 à 3 du projet d'article 3 n'exige pas que l'auteur soit un responsable ou un agent de l'État ${ }^{48}$.

Bien d'autres développements ont été apportés en commentaires à ce qu'il faudra entendre, dans le cadre du projet d'article 3, par «attaque généralisée ou systématique ${ }^{49}$; [ « lancée contre toute population civile » et «en application d'une politique d'État ou d'organisation $\left.{ }^{50} »\right]$; « en connaissance de cette attaque ${ }^{51} »$.

Après avoir posé formellement que les crimes contre l'humanité sont des crimes de droit international, le texte des projets d'articles relève clairement une obligation des États de les prévenir et de les punir. Seulement, à l'analyse, il sera démontré que cette dernière obligation de "punir» ne paraît que trompeuse. L'obligation n'étant en réalité que celle de poursuivre tout court.

\section{L'obligation de prévenir les crimes contre l'humanité dans les projets d'articles}

Généralement, les traités portant sur les mesures prises pour ériger en infraction certains actes, à l'instar des graves violations des droits de l'homme,

46 La CDI a tenu toutefois à préciser que les éléments adoptés dans le cadre d'une loi nationale, qui n'entreraient pas dans le champ d'application du présent projet d'articles, ne bénéficieraient pas des dispositions prévues par celui-ci, notamment en matière d'extradition et d'entraide judiciaire (voir Ibid, commentaire 41 à la $\mathrm{p} 78$ ).

47 Ibid, commentaires 1-41 aux pp 61-78.

48 Ibid, commentaire 31 à la p 74.

49 Ibid, commentaires 10-16 aux pp 64-67.

50 Ibid, commentaires 17-33 aux pp 67-75.

51 Ibid, commentaires 34-36 aux pp 75-76. 
portent essentiellement sur la punition des auteurs des crimes visés, mais beaucoup mentionnent également l'une ou l'autre forme d'obligation pour les États parties de prendre des mesures de prévention. Cette obligation de prévention peut être énoncée dans un seul article traitant la question de la prévention au sens large ou figurer dans plusieurs articles qui, pris ensemble, servent le même propos ${ }^{52}$. Les États se voient ainsi imposer une obligation de prévenir la réalisation d'un fait, apprécié au sens large, susceptible de pouvoir constituer une violation de la norme dont la convention $\mathrm{a}$, de manière très générale, l'objectif de protéger ${ }^{53}$. Les obligations de prévention de l'État les plus nombreuses et les plus importantes sont qualifiées d'obligations de due diligence. Ceci résulte du fait qu'une large partie des activités de prévention imposées aux autorités étatiques dans certaines circonstances concrètes ne sont pas, de par leur nature, propres à garantir un résultat certain, mais elles sont soumises à des circonstances aléatoires. Généralement dans les activités de prévention, l'obligation de l'État est seulement une obligation de s'efforcer, avec due diligence, d'atteindre un but $^{54}$.

Dans le cadre du texte des projets d'articles sur les crimes contre l'humanité, l'obligation de prévention est prévue par les articles 2 et 4 . D'une part, le projet d'article 2 énonce une obligation générale en ce sens que les crimes contre l'humanité, qu'ils soient ou non commis en temps de conflit armé, sont des crimes au regard du droit international que les États s'engagent à prévenir et à punir. D'autre part, le projet d'article 4 consacre l'obligation pour l'État partie de prendre des mesures spéciales, législatives, administratives, judiciaires ou autres mesures efficaces de prévention dans tout territoire sous sa juridiction ou son contrôle ainsi que coopérer avec les autres États, les organisations intergouvernementales pertinentes et, selon qu'il convient, d'autres organisations, pour prévenir les crimes contre l'humanité ${ }^{55}$.

L'obligation générale figurant au projet d'article 2 s'applique aussi bien à l'obligation de prévenir les crimes contre l'humanité qu'à celle d'en punir les auteurs. Certes,

[1]'une des manières les plus efficaces de prévenir la commission d'actes criminels, en général, est de prévoir des sanctions pénales à l'encontre des personnes qui viendraient à commettre de tels actes, et d'appliquer effectivement ces sanctions à ceux qui auraient commis les actes dont on cherche à éviter le renouvellement ${ }^{56}$,

52 Voir Murphy, Premier rapport sur les crimes contre l'humanité, supra note 11.

53 Sébastien Touze, «La notion de prévention en droit international des droits de l'homme » dans Emmanuel Decaux et Sébastien Touze, dir, La prévention des violations des droits de l'Homme, Paris, Pedone, 2015 à la p 19.

54 Riccardo Pisillo Mazzeschi, « Responsabilité de l'État pour violation des obligations positives relatives aux droits de l'homme» (2008) 33 Rec des Cours 175 à la p 334.

55 Voir Projets d'articles sur les crimes contre l'humanité, supra note 11, art 4.

56 Application de la convention pour la prévention et la répression du crime de génocide (BosnieHerzégovine c Serbie-et-Monténégro), arrêt du 26 février 2007, [2007] CIJ rec au para 426 [Affaire du génocide bosniaque]. 
mais les deux obligations de prévenir et de punir demeurent distinctes quoique reliées, comme cela sera démontré dans les lignes qui suivent. Une lecture préliminaire révèle que le projet d'article 2 semble calqué sur le modèle ${ }^{57}$ de l'article I de la Convention sur le génocide de $1948^{58}$. Par contre, le projet d'article 4 semble ressortir une différence entre le texte provisoirement adopté et la Convention sur le génocide. En effet, tant l'article V de la Convention sur le génocide que ce projet d'article 4 prévoient l'adoption d'une «législation nécessaire» pour donner effet à leurs dispositions. Mais le projet d'article 4 prévoit en outre que l'État partie prendra, dans tout territoire sous sa juridiction ou son contrôle, des mesures spéciales, législatives, administratives, judiciaires ou autres mesures efficaces de prévention. Il prévoit aussi que les États sont tenus de coopérer entre eux, avec les organisations intergouvernementales pertinentes et, selon qu'il convient, d'autres organisations pour cette même fin. Ce qui est aussi plus large que le mécanisme prévu par l'article VIII de la Convention sur le génocide, aux termes duquel

[t]oute Partie contractante peut saisir les organes compétents de l'Organisation des Nations Unies afin que ceux-ci prennent, conformément à la Charte des Nations Unies, les mesures qu'ils jugent appropriées pour la prévention et la répression des actes de génocide ou de l'un quelconque des autres actes énumérés à l'article III.

Pour mieux cerner la portée des obligations définies par les projets d'articles 2 et 4, il est important d'analyser les libellés de ces dispositions - en particulier, le caractère obligatoire de l'engagement de prévenir les crimes contre l'humanité (A) et la portée normative de cette obligation (B).

\section{A. Caractère obligatoire de l'engagement de prévenir les crimes contre l'humanité}

Les projets d'articles 2 et 4 du texte provisoirement adoptés ne seront pas les seules dispositions d'un instrument international à prévoir l'obligation pour les États parties de prendre certaines mesures afin de prévenir les actes qu'il vise à interdire. Bien d'autres instruments comportent une obligation similaire, sous des formes diverses. Il en va ainsi de la Convention sur le génocide du 9 décembre 1948 (art. I); de la Convention contre la torture et autres peines et traitements cruels, inhumains ou dégradants $^{59}$ du 10 décembre 1984 (art 2); de la Convention sur la prévention et la

57 Voir Murphy, Premier rapport sur les crimes contre l'humanité, supra note 11 au para 112: « la rédaction de l'article premier de la Convention sur le génocide - « confirmant » que le génocide est un crime du droit des gens et engageant les États parties à le prévenir et à le punir - reste un modèle utile pour formuler une obligation générale dans une convention tendant à prévenir les crimes contre l'humanité ».

58 Convention sur le génocide, supra note 5, art I: «[1]es Parties contractantes confirment que le génocide, qu'il soit commis en temps de paix ou en temps de guerre, est un crime du droit des gens, qu'elles s'engagent à prévenir et à punir ».

59 Convention contre la torture et autres peines ou traitements cruels, inhumains ou dégradants, 10 décembre 1984, 1465 RTNU 85, RT Can 1987 n 36 (entrée en vigueur : 26 juin 1987) [Convention contre la torture]. 
répression des infractions contre les personnes jouissant d'une protection internationale, y compris les agents diplomatiques du 14 décembre 1973 (art 4); de la Convention sur la sécurité du personnel des Nations Unies et du personnel associé du 9 décembre 1994 (art 11); de la Convention internationale pour la répression des attentats terroristes à l'explosif du 15 décembre 1997 (art 15).

À cet égard, on pourrait essayer de comprendre aussi bien le caractère obligatoire que la portée normative de l'engagement de prévenir les crimes contre l'humanité qui est énoncé aux projets d'articles 2 et 4 à l'aune de développements qui ressortent des autres instruments prévoyant des dispositions similaires. Mais cette méthodologie appelle une certaine prudence. Le contenu de l'obligation de prévention varie, en effet, d'un instrument à l'autre, selon le libellé des dispositions pertinentes et en fonction de la nature même des actes qu'il s'agit de prévenir. Telle est l'affirmation de la Cour internationale de justice (Cour ou CIJ) à l'occasion de l'Affaire relative à l'application de la convention pour la prévention et la répression du crime de génocide (Bosnie-Herzégovine c Serbie-et-Monténégro) ${ }^{60}$. La Cour a, par ailleurs, avisé «[qu'elle] n'entend[ait] donc pas établir par sa décision une jurisprudence générale qui serait applicable à tous les cas où un instrument conventionnel, ou toute autre norme obligatoire, comporte, à la charge des États, une obligation de prévenir certains actes ${ }^{61} »$. Mais dans la mesure où la CDI s'est expressément inspirée de la Convention sur le génocide quant à l'obligation générale de prévention contenue particulièrement au projet d'article 2 , l'on pourrait se référer à l'analyse du contenu faite par la Cour de l'engagement de prévenir le génocide afin de dégager le caractère obligatoire de celui de prévenir les crimes contre l'humanité ${ }^{2}$.

En effet, les termes «s'engagent à » qui ressortent clairement des projets d'articles 2 et 4 restent adéquats aux fins d'analyse du caractère obligatoire de cet engagement. Lu à la lumière de l'interprétation de la Cour dans l'Affaire du génocide bosniaque: "[e]n son sens ordinaire, le terme "s'engagent" signifie promettre formellement, s'obliger, faire un serment ou une promesse, convenir, accepter une obligation $^{63} »$. Examinant la demande en indication de mesures conservatoires, la Cour a estimé que l'«engagement de prévenir» visé à l'article premier de la Convention sur le génocide imposait aux deux parties «l'incontestable obligation de faire tout ce qui [était] en leur pouvoir pour [...] assurer la prévention [de tels actes] à $l^{\prime}$ avenir $^{64} »$. Lors de l'examen au fond, elle a décrit cet engagement comme une promesse formelle «qui ne revêt pas un caractère purement incitatif et ne se limite pas à l'énoncé d'une finalité [...] et ne doit pas être interprété[e] comme une simple

60 Affaire du génocide bosniaque, supra note 56 au para 429.

61 Ibid.

62 Murphy, Premier rapport sur les crimes contre l'humanité, supra note 11 : «Au surplus, l'utilisation de cette formulation permettrait d'harmoniser l'actuel projet d'articles avec une convention largement ratifiée relative à un autre crime grave de droit international (en janvier 2015, 146 États étaient parties à la Convention sur le génocide) ».

63 Affaire du génocide bosniaque, supra note 56 au para 162.

64 Application de la convention pour la prévention et la répression du crime de génocide (BosnieHerzégovine c Serbie-et-Monténégro), Ordonnance en indication des mesures conservatoires du 8 avril 1993, [1993], CIJ rec au para 45. 
introduction aux dispositions qui évoquent ensuite expressément les mesures législatives, les poursuites et l'extradition ${ }^{65}$ ». De l'entendement de la Cour, l'analyse des travaux préparatoires permet de dire que l'article premier de la Convention sur le génocide "impose effectivement des obligations distinctes en sus de celles édictées par d'autres articles de la Convention»; et qu' "en particulier, les parties contractantes ont directement l'obligation de prévenir le génocide ${ }^{66} »$. Construit sur le même modèle, l'engagement de prévenir les crimes contre l'humanité, tel qu'énoncé au projet d'article 2 et au paragraphe 1 du projet d'article 4 , est destiné à exprimer le même effet juridique; il ne revêt pas, lui non plus, un caractère purement incitatif et il ne se limite pas à l'énoncé d'une finalité, pas plus qu'il n'est simplement une introduction à la suite des projets d'articles ${ }^{67}$. Toutefois, la spécificité de l'article premier de la Convention sur le génocide a ainsi forgé sa lecture autonome et suffit à considérer que les États ont une obligation à portée générale-notamment extraterritoriale - de prévenir le crime de génocide ${ }^{68}$. Cette interprétation pourrait ne pas être la même s'agissant des crimes contre l'humanité. D'abord, l'on ne pourrait pas soutenir une lecture autonome du projet d'article 2 sur les crimes contre l'humanité comme il en est de l'article I de la Convention sur le génocide. Le commentaire 1) apporté par la CDI au projet d'article 2 est plus explicite à ce sujet :

[1]e projet d'article 2 énonce l'obligation générale qu'ont les États de prévenir les crimes contre l'humanité et d'en punir les auteurs. La teneur de cette obligation générale sera examinée à travers les diverses obligations plus spécifiques énoncées dans les projets d'article suivants, à partir du projet d'article 4 . Ces obligations spécifiques porteront sur les mesures que les États doivent prendre dans leurs systèmes juridiques nationaux, ainsi que sur leur coopération avec d'autres États, les organisations intergouvernementales pertinentes et, s'il y a lieu, d'autres organisations ${ }^{69}$.

Ensuite, il semble, en effet, que la CDI ait voulu réduire la sphère d'action de l'État au «territoire sous sa juridiction ou son contrôle » et partant, la portée de l'obligation de prévenir ne serait plus la même que dans la Convention sur le génocide. Ce qui amène à analyser la portée normative de cette obligation de prévenir le génocide.

65 Murphy, Premier rapport sur les crimes contre l'humanité, supra note 11.

66 Ibid aux paras 162, 165.

67 Voir à cet effet le $7^{\mathrm{e}}$ commentaire du projet d'article 4. Projets d'articles sur les crimes contre l'humanité, supra note 11 à la $\mathrm{p} 83$.

68 Vanessa Thalmann, « National Criminal Jurisdiction over Genocide » dans Paola Gaeta, The UN Genocide Convention - A commentary, New York, Oxford University Press, 2009 à la p 233: « Article I does not contain any territorial limitation. " Dans l'affaire du génocide bosniaque, la Cour a également jugé que les obligations matérielles découlant de cet article ne semblaient pas être territorialement limitées, mais, au contraire, «s'appliqu[ai]ent à un État, où que celui-ci se trouve agir ou en mesure d'agir pour s'acquitter des obligations en question » (Affaire du génocide bosniaque, supra note 56 au para 183).

69 Murphy, Premier rapport sur les crimes contre l'humanité, supra note 11 à la p 56. 


\section{B. La portée normative de l'obligation de prévenir les crimes contre l'humanité}

La portée des obligations positives des États de prévenir les crimes contre l'humanité en vertu du texte des projets d'articles est substantiellement régie par l'article 4, aux termes duquel ces obligations doivent être observées dans le «territoire sous la juridiction ou [le] contrôle » de l'État. L'on sait à cet égard que l'utilisation des expressions " territoire » sous sa "juridiction » ou son " contrôle » dans une disposition de cette nature n'est pas fortuite et a certainement pour effet de délimiter le champ opératoire des obligations positives des États de prévenir les crimes définis au projet d'article $3^{70}$. Dans plusieurs conventions relatives aux droits de l'homme qui comportent des dispositions similaires à celles du projet d'article 4, ces expressions servent à définir la portée territoriale des obligations des États en vertu du traité ${ }^{71}$. Ce projet d'article, comme il en est des autres dispositions conventionnelles similaires, fait en partie écho à l'article 29 de la Convention de Vienne sur le droit des traités entre États qui énonce qu'à moins «qu'une intention différente ne ressorte du traité ou ne soit par ailleurs établie, un traité lie chacune des parties à l'égard de l'ensemble de son territoire ». Certains commentateurs trouvent par exemple en la notion de "juridiction » l'exercice d'une compétence nationale territoriale alors que d'autres y trouvent le fondement d'une compétence extraterritoriale $^{72}$. Dès lors, compte tenu du débat sur l'interprétation des termes « territoire », « juridiction » et « contrôle », il convient d'explorer ce projet d'article 4 afin de voir si son libellé permet de ressortir clairement des indices sur leur signification et leur portée possibles. Mais parce que le terme «territoire »-ici entendu en son sens le plus habituel comme l'espace géographique sur lequel un État exerce l'intégralité de ses compétences ou certaines compétences déterminées ${ }^{73}-$ ne semble pas poser de problème d'interprétation, seuls les termes «juridiction » et « contrôle » méritent d'être analysés.

70 Ces termes ont été interprétés dans le cadre de traités de droits de l'homme par des organes internationaux, régionaux et nationaux de contrôle comme ayant eu un impact majeur sur leur applicabilité extraterritoriale. Pour en savoir plus, voir Michal Gondek, The Reach of Human Rights in a Globalising World: Extraterritorial Application of Human Rights Treaties, Antwerp, Intersentia, 2009 aux pp 126-31, 367 [Gondek].

71 Voir Ludovic Hennebel et Hélène Tigroudja, dir, Traité de Droit international des droits de l'homme, Paris, Pedone, 2016 à la p 674 : « À l'exception du PIDESC et de la Charte africaine des droits de l'homme et des peuples - qui ne le précisent pas explicitement -, les conventions générales limitent la portée des obligations conventionnelle des États Parties aux personnes qui sont sous leur juridiction comme le montrent l'article $1^{\text {er }}$ de la $\mathrm{CEDH}$ ("toute personne relevant de leur juridiction"); l'article $1^{\text {er }}$ de la CADH ("toute personne relevant de leur compétence"); l'article $2 \S 1$ du PIDCP ("tous les individus se trouvant sur leur territoire et relevant de leur compétence"); l'article 3 de la Charte arabe des droits de l'homme ("tout individu relevant de sa juridiction") ». Dans la même idée, voir María Luisa Piqué, «Beyond Territory, Jurisdiction, and Control: Towards a Comprehensive Obligation to Prevent Crimes Against Humanity » dans Morten Bergsmo et Song Tianying, dir, On the Proposed Crimes Against Humanity Convention, Bruxelles, Torkel Opsahl Academic EPublisher, 2014 à la p 147 [Piqué].

72 À propos de cette controverse, voir notamment Karen da Costa, The extraterritorial application of selected human rights treaties, vol 11, Leiden, Boston, Martinus Nijhoff Publishers, 2013 aux pp 9-12; Guillaume Grisel, Application extraterritoriale $d u$ droit international des droits de l'homme, Collection de droit international public, Bâle, Helbing Lichtenhahn, 2010 [Grisel].

73 Jean Salmon, dir, Dictionnaire de droit international public, Bruxelles, Bruylant, 2001 à la p 1076. 
En effet, l'interprétation de «juridiction» et de «contrôle » prévus à l'article 4 appelle diverses significations. L'on ne peut que constater, au regard du $6^{\mathrm{e}}$ commentaire apporté par la CDI au projet d'article 6 relatif à l'établissement de la compétence, qu'elle se propose de réexaminer à l'avenir le texte du projet d'article 4 à des fins de cohérence terminologique en ce qui concerne les termes « territoire sous sa juridiction ou son contrôle ». En attendant cet éclaircissement, il n'est pas inutile de suggérer un sens à ces expressions. En droit international général, la juridiction est souvent comprise soit comme la compétence elle-même, soit l'ensemble des compétences de l'État, c'est-à-dire «le pouvoir juridique conféré ou reconnu par le droit international à un État de soumettre des personnes, physiques ou morales, des biens et des activités à son ordre juridique ${ }^{74} \gg$. Les compétences de l'État sont essentiellement territoriales et exceptionnellement extraterritoriales. Il en va de même pour sa juridiction ${ }^{75}$. La Cour européenne des droits de l'homme semble avoir choisi cette interprétation de la notion de juridiction dans sa décision Bankovic de $2001^{76}$. Elle n'est toutefois pas la seule interprétation possible. La juridiction de l'État pourrait signifier autre chose que la simple compétence de l'État. À partir de cette hypothèse, deux voies sont ouvertes : d'une part, la «juridiction » du projet d'article 4 sous examen serait uniquement l'espace dans lequel l'État est habilité à exercer ses compétences, c'est-à-dire son territoire souverain, et exceptionnellement d'autres territoires (occupation militaire, cession à bail, etc.) ${ }^{77}$. Mais cette lecture qui tend à assimiler la juridiction au territoire, même si les deux termes ne sont pas synonymes, ne peut être soutenue en l'espèce. Le fait que la CDI ait choisi l'expression « territoire sous sa juridiction » ne permet plus d'envisager la juridiction comme un territoire pour la simple raison que ceci serait une redondance. En présence de deux lectures possibles d'un texte, il paraît raisonnable de rejeter celle qui aboutit à une redondance au profit de celle qui confère une pleine portée à chaque mot $^{78}$. D'autre part, la notion de juridiction aurait un sens plus proche de celui qu'elle a généralement dans les conventions relatives aux droits de l'homme, où des dispositions semblables sont très courantes $^{79}$. Selon ce dernier point de vue, la « juridiction » ne doit pas être comprise

74 Ibid aux pp 210 et suiv, 625. Selon un rapport de la CDI de 1994, « La juridiction d'un État englobe, outre les activités entreprises à l'intérieur du territoire de cet État, les activités sur lesquelles il est autorisé à exercer sa compétence et son autorité », ACDI, 1994-II/2 à la p 172 au para 15.

75 Conséquences juridiques de l'édification d'un mur dans le territoire palestinien occupé, Avis consultatif du 9 juillet 2004, [2004] CIJ rec au para 109 : «La Cour observera que, si la compétence des États est avant tout territoriale, elle peut parfois s'exercer hors du territoire national ».

76 Bankovic et autres c Belgique et autres, $n^{\circ}$ 52207/99, [2001] XII CEDH 361, au para 59 : « En ce qui concerne les 'sens ordinaire' des termes pertinents figurant dans l'article 1 de la Convention, la Cour considère que, du point de vue du droit international public, la compétence juridictionnelle d'un État est principalement territoriale. Si le droit international n'exclut pas un exercice extraterritorial de sa juridiction par un État, les éléments ordinairement cités pour fonder pareil exercice (nationalité, pavillon, relations diplomatiques et consulaires, effets, protection, personnalité passive et universalité, notamment) sont en règle générale définis et limités par les droits territoriaux souverains des autres États concernés ».

77 Grisel, supra note 72 à la p 51.

78 Voir par exemple Us vs Pan, Award of 27 June 1993, 7 Ann Dig (1993-34), 225, 257 (Us-pan Claims Comm's) cité dans Grisel, supra note 72 à la p 61.

79 Pour une vue de toutes les utilisations du mot «juridiction» dans les traités relatifs aux droits de l'homme, voir par exemple, Marko Milanović, «From Compromise to Principle: Clarifying the Concept of State Jurisdiction in Human Rights Treaties » (2008) 8:3 Human Rights Law Review 411. 
comme telle en droit international général mais comme se référant «à un type particulier de pouvoir factuel, d'autorité ou de contrôle qu'un État a sur un territoire et par conséquent, sur des personnes sur ce territoire ${ }^{80} \gg$. La juridiction serait dans ce cas une notion plus large que celle de compétence puisqu'elle inclurait également l'exercice d'autorité de pur fait qui servirait ainsi de condition pour évaluer l'existence d'une obligation particulière d'un État concernant une victime particulière - ou une victime potentielle - d'une violation des droits de l'homme en raison de sa présence sur un certain territoire ${ }^{81}$. C'est ainsi que le Comité contre la torture interprète, dans son observation générale $n^{\circ} 2^{82}$, le paragraphe 1 de l'article 2 de la Convention contre la torture qui, par ailleurs, a inspiré la rédaction du projet d'article 4 sur les crimes contre l'humanité comme le relève la CDI au commentaire $13^{83}$. C'est ainsi que l'ancienne Commission européenne des droits de l'homme lisait l'article $1^{\mathrm{er}}$ de la CEDH dans la décision Chypre c Turquie de $1975^{84}$. La même interprétation a été choisie par le Comité des droits de l'homme dans son observation générale $n^{\circ} 31$ où il comprend la notion de juridiction comme signifiant qu' « un État partie doit respecter et garantir à quiconque se trouve sous son pouvoir ou son contrôle effectif les droits reconnus dans le Pacte même s'il ne se trouve pas sur son territoire ${ }^{85}$ ». Toutefois, à

$80 \quad$ Ibid à la p 428.

81 Grisel, supra note 72; Gondek, supra note 70 à la p 168.

82 Comité contre la torture, 24 janvier 2008, Observation générale $n^{\circ} 2$, (Application de l'article 2 par les États parties), Doc off AG NU, (2008) Doc NU CAT/C/GC/2 au para 16: «[1]e paragraphe 1 de l'article 2 impose à tout État partie de prendre des mesures efficaces pour empêcher que des actes de torture ne soient commis non seulement sur son propre territoire mais aussi dans tout territoire sous sa juridiction. Le Comité considère que le 'territoire' s'étend à toutes les régions sur lesquelles l'État partie exerce de fait ou de droit, directement ou indirectement, en tout ou en partie, un contrôle effectif, conformément au droit international. [...] Le Comité considère également que la notion de 'territoire' à l'article 2 doit s'étendre en outre aux situations dans lesquelles l'État partie exerce, directement ou indirectement, de fait ou de droit, un contrôle sur des détenus ». En ce sens, voir aussi Manfred Nowak et Elizabeth McArthur, The United Nations Convention Against Torture: A Commentary, Oxford, Oxford University Press, 2008 aux pp 116-17.

83 « Troisièmement, et en conséquence de ce qui précède, l'engagement énoncé au paragraphe 1 du projet d'article 4 oblige les États à prendre activement et en amont des mesures destinées à contribuer à empêcher l'infraction d'être commise, par exemple au moyen de 'mesures législatives, administrative, judiciaires et autres mesures efficaces de prévention dans tout territoire sous sa juridiction ou son contrôle', comme indiqué à l'alinéa a). Ce texte est inspiré du paragraphe 1 de l'article 2 de la Convention contre la torture, qui dispose ce qui suit : 'Tout État partie prend des mesures législatives, administratives, judiciaires et autres mesures efficaces pour empêcher que des actes de torture soient commis dans tout territoire sous sa juridiction' ». Murphy, Premier rapport sur les crimes contre l'humanité, supra note 11 aux pp 85-86.

84 Commission européenne des droits de l'homme, Chypre c Turquie, requêtes n ${ }^{\circ}$ 6780/74 et 6950/75, 26 mai 1975, (1975) 18:1 Ann Conv eur DH aux pp 118-120 [Chypre c Turquie]: «à l'article 1 de la Convention, les Hautes Parties contractantes reconnaissent les droits et les libertés au Titre I à toute personne relevant de leur juridiction. La Commission estime que ce terme ne se limite pas, comme le déclare la Gouvernement défendeur, au seul territoire national des Hautes Parties contractantes en cause. Il ressort du libellé, notamment de la version française, et de l'objet dudit article que du but de la Convention tout entière que les Hautes Parties contractantes sont tenues d'assurer ces droits et libertés à toute personne relevant effectivement de leur autorité et de leur responsabilité, que cette autorité s'exerce sur le territoire ou à l'étranger ».

85 Comité des droits de l'homme, Observation générale $n^{\circ} 31$, La nature de l'obligation juridique générale imposée aux États parties au Pacte, Doc off CES NU, (2004), Doc NU CCPR/C/21/Rev.1/Add.13 (2004) au para 10. 
propos de cette dernière hypothèse, l'on peut constater que la logique du projet d'article 4 ne permet pas de conférer une telle portée à l'obligation de prévenir les crimes contre l'humanité car, non seulement, la « juridiction» du projet d'article 4 ne peut être assimilée au "territoire » comme il a été précédemment noté, mais aussi, elle ne peut être comprise comme ce type particulier de pouvoir factuel, d'autorité ou de contrôle qu'un État a sur un territoire ou sur des personnes sur ce territoire. Autrement, il n'aurait pas été nécessaire que la CDI ajoute les mots « territoire sous son contrôle » parce que le « territoire sous la juridiction », dans l'hypothèse discutée, implique déjà l'exercice d'un pouvoir de fait, d'autorité ou de contrôle sur une personne ou un territoire. Et donc, l'expression «territoire sous son contrôle » serait dépourvue de tout effet utile. Pourtant, de la lecture du projet d'article 4, il appert que la "juridiction » est utilisée comme une alternative au "contrôle », la conjonction «ou» reliant les deux alternatives. Ce qui laisse entrevoir que les rédacteurs pensaient à deux situations différentes et mutuellement exclusives: un certain territoire est sous la juridiction d'un État et un certain territoire est sous son contrôle ${ }^{86}$.

Le «contrôle» est aussi un concept délicat. Il a suscité plusieurs interprétations en droit international et ce, dans plusieurs chapitres différents ${ }^{87}$. Dans le projet d'article 4, il apparaît comme un critère pour déterminer si un État a l'obligation de prévenir les crimes contre l'humanité résultant des actes qui dépassent sa juridiction. L'utilisation du terme "contrôle» n'est pas inhabituelle dans la jurisprudence internationale relative aux droits de l'homme. Mais, comme on peut le constater à travers les précédentes lignes, elle a servi de critère pour déterminer la « juridiction» de l'État sur les territoires où des violations des droits de l'homme ont eu lieu ou sur des victimes de ces violations. Selon cette interprétation, le « contrôle » n'est pas un concept autonome concernant les limites des obligations des États mais plutôt une condition nécessaire pour prouver l'existence de la «juridiction ${ }^{88}$ ». C'est suivant cette interprétation que la Cour européenne des droits de l'homme a utilisé le critère du «contrôle effectif» pour déterminer si un État partie à la Convention était sous l'obligation d'assurer les droits et libertés de la CEDH dans un territoire occupé ${ }^{89}$.

86 Dans le même sens, mais à propos de l'ancien article $8(1)$ du projet de Convention, devenu après plusieurs modifications, l'actuel projet d'article 4. (Piqué, supra note 71 à la p 151). Bien plus, le commentaire 18) de la CDI laisse entrevoir cette différence en ce termes : « Comme la Commission l'a déjà expliqué, [cette formulation] s'applique à des situations où un État exerce une juridiction de facto, même lorsqu'il n'a pas de juridiction de jure, par exemple les situations d'intervention, d'occupation et d'annexion illicites au regard du droit international. On peut se référer, à cet égard, à l'avis consultatif de la CIJ relatif à l'Affaire de la Namibie (Sud-Ouest africain). Dans cet avis, la Cour, après avoir estimé l'Afrique du Sud responsable d'avoir créé et prolongé une situation dont la Cour avait constaté l'illicéité et avoir conclu que l'Afrique du Sud était dans l'obligation de retirer son administration de la Namibie, a néanmoins assigné certaines conséquences juridiques au contrôle de facto exercé sur celleci par l'Afrique du Sud ». Murphy, Premier rapport sur les crimes contre l'humanité, supra note 11 à la p 88.

87 Pour une vue récente du critère de contrôle, voir Gionavi Distefano et Aymeric Chêche, « L'organe de facto dans la responsabilité internationale » (2015) 15 AFDI aux pp 19 et suiv [Distefano et Chêche].

88 Dans le même sens, Piqué, supra note 71 à la p 155.

89 Loizidou c Turquie (Exceptions préliminaires) (1995), 310 CEDH (Sér A) 7, para 62, 20 EHRR 99 [Loizidou]. Mais faut-il préciser que le critère de " contrôle effectif » a été utilisé pour la première fois par la CIJ, dans l'Affaire des activités militaires et paramilitaires au Nicaragua et contre celui-ci 
En l'occurrence, elle a appliqué ce critère aux violations des droits de l'homme à Chypre et a constaté que la Turquie exerçait effectivement un « contrôle effectif », qui pourrait être exercé « directement, par l'intermédiaire de ses forces armées ou par une administration locale subordonnée ${ }^{90} »$. Dans sa décision au fond, la Cour a maintenu cette position, bien qu'elle ait légèrement changé le critère en "contrôle général effectif $f^{91}$ ». En même temps, le « contrôle » a également servi de critère d'attribution à l'État d'un fait illicite en vertu des règles de la responsabilité des États ${ }^{92}$. Il s'agit ici d'un examen de la responsabilité de l'État pour des faits accomplis par des particuliers - organes de jure ou de facto - sur un territoire (sans juridiction), en se servant ainsi du critère de « contrôle » pour déterminer le seuil de dépendance de ces particuliers envers cet État et partant, la capacité d'influence de ce dernier sur ce territoire. C'est suivant ce sens que le concept de « contrôle » s'est progressivement et diversement forgé par la voie juridictionnelle à travers - sans qu'il soit besoin d'en dire plus - l'Affaire des otages ${ }^{93}$ (1980); l'Affaire Nicaragua ${ }^{94}$ (1986); l'Affaire Tadic $^{95}$ (1999) et enfin la toute dernière affaire en date, l'Affaire Génocide ${ }^{96}$ (2007).

Quoi qu'il en soit, l'ajout de « contrôle » devient un moyen d'élargir la portée des obligations positives contenues au projet d'article 4. En même temps, cependant, cette interprétation implique que - dans le texte du projet d'article sur les crimes contre l'humanité - la «juridiction » est employée dans son sens que lui confère le

(Nicaragua c États Unis), arrêt du 27 juin 1986, [1986] CIJ rec au para 115 [Activités militaires et paramilitaires].

90 Ibid. Cette interprétation a été confirmée en 2011 par l'arrêt Al-Skeini et autres c Royaume-Uni [n ${ }^{\circ} 55721 / 07$, (2011) VII CEDH 199, 53 EHRR 18] rendu par la grande chambre en date du 7 juillet 2011. La Cour européenne des droits de l'homme a énoncé au paragraphe 138 que «le principe voulant que la juridiction de l'État contractant au sens de l'article $1^{\text {er }}$ soit limitée à son propre territoire connaît une autre exception lorsque, par suite d'une action militaire (légale ou non), l'État exerce un contrôle effectif sur une zone située en dehors de son territoire. L'obligation d'assurer dans une telle zone le respect des droits et libertés garantis par la Convention découle du fait de ce contrôle, qu'il exerce directement, par l'intermédiaire des forces armées de l'État ou par le biais d'une administration locale subordonnée [...] Dès lors qu'une telle mainmise sur un territoire est établie, il n'est pas nécessaire de déterminer si l'État contractant qui la détient exerce un contrôle précis sur les politiques et actions de l'administration locale qui lui est subordonnée. Du fait qu'il assure la survie de cette administration grâce à son soutien militaire et autre, cet État engage sa responsabilité à raison des politiques et actions entreprises par elle. L'article $1^{\text {er }}$ lui fait obligation de reconnaître sur le territoire en question la totalité des droits matériels énoncés dans la Convention et dans les protocoles additionnels qu'il a ratifiés, et les violations de ces droits lui sont imputables ».

91 Loizidou c Turquie (Arrêt) (1996), 310 CEDH (Sér A) 29, 20 EHRR 136.

92 Comme le relèvent Gionavi Distefano et Aymeric Chêche (Distefano et Chêche, supra note 89 à la p 4), ces règles sont à ce jour codifiées par un ensemble d'articles adoptés en 2001 par la CDI et considérés par les États, la jurisprudence ainsi que la doctrine comme 'le reflet fidèle à bien des égards' du droit international en matière de responsabilité internationale (Affaire du génocide bosniaque, supra note 56 au para 401 : " Ainsi se présente aujourd'hui le droit international coutumier en la matière, tel que reflété par es articles de la CDI sur la responsabilité des États »).

93 Personnel diplomatique et consulaire des États-Unis à Téhéran (États-Unis c Iran), Arrêt du 24 mai 1980, [1980] CIJ rec aux paras 58-74 [Personnel diplomatique et consulaire].

94 Activités militaires et paramilitaires, supra note 89 aux paras 99-116.

95 Le Procureur c Duško Tadić, IT-94-1-T (7 mai 1997) (Tribunal pénal international pour l'exYougoslavie, Chambre de première instance); Le Procureur c Duško Tadić, IT-94-1-T (15 juillet 1999) aux paras 87-151 (Tribunal pénal international pour l'ex-Yougoslavie, Chambre d'appel) [Tadić].

96 Affaire du génocide bosniaque, supra note 56 aux paras 392 et suiv. 
droit international général. Par conséquent, afin de déterminer si les États sont tenus de prévenir les crimes contre l'humanité en vertu du texte provisoirement adopté, la «juridiction » prévue à l'article 4 devrait, en définitive, être interprétée suivant son sens en droit international général - de «compétence », c'est-à-dire le titre légal à agir conféré par une norme de droit international coutumier ou conventionnel ${ }^{97}-$ et exclusivement sur une base territoriale parce que la disposition se réfère à « territoire sous la juridiction ou son contrôle ». Toujours est-il que la mention explicite du territoire suggère qu'il convient d'accorder à celui-ci une plus grande importance que ne le font d'autres instruments dépourvus d'une telle mention, comme le relève Dominic McGoldrick ${ }^{98}$. Le terme "contrôle » est, à cet effet, censé englober les situations dans lesquelles les États exercent une sorte de pouvoir de fait sur un territoire sans aucune compétence légale ${ }^{99}$. C'est à ce titre que la portée normative de l'obligation de prévenir les crimes contre l'humanité pourrait atteindre des situations où un État exerce une juridiction de facto, même lorsqu'il n'a pas de juridiction de jure. Il en est ainsi notamment dans les situations d'intervention, d'occupation et d'annexion illicites au regard du droit international ${ }^{100}$. Et vice versa, dans les cas où, bien que l'État ait une juridiction de jure sur un territoire, il n'a pas le contrôle des situations qui s'y passent ${ }^{101}$.

97 Voir Grisel, supra note 72 à la p 137.

98 Dominic McGoldrick, «Extraterritorial Application of the International Covenant on Civil and Political Rights » dans Fons Coomans et Menno Kamminga, dir, Extraterritorial Application of Human Rights Treaties, Antwerp, Oxford, Intersentia 2004 à la p 48.

99 Chypre c Turquie, supra note 84 à la p 154.

100 Le « contrôle sans juridiction » pourrait se produire dans des situations telles que celle représentée par la CIJ dans l'Affaire des activités armées sur le territoire du Congo (République Démocratique du Congo c Ouganda) arrêt, [2005] CIJ rec au para 178 : « La Cour conclut ainsi que l'Ouganda était une puissance occupante dans le district de l'Ituri à l'époque pertinente. En tant que tel, il se trouvait dans l'obligation, énoncée à l'article 43 du règlement de La Haye de 1907, de prendre toutes les mesures qui dépendaient de lui en vue de rétablir et d'assurer, autant qu'il était possible, l'ordre public et la sécurité dans le territoire occupé en respectant, sauf empêchement absolu, les lois en vigueur en RDC. Cette obligation comprend le devoir de veiller au respect des règles applicables du droit international relatif aux droits de l'homme et du droit international humanitaire, de protéger les habitants du territoire occupé contre les actes de violence et de ne pas tolérer de tels actes de la part d'une quelconque tierce partie ».

101 La «juridiction sans contrôle» pourrait se produire dans des situations telles que celle décrite par la CEDH dans son arrêt rendu à l'occasion de l'affaire Ilascu et autres c Moldavie et Russie, $\mathrm{n}^{\circ}$ 48787/99, [2004] VII CEDH, para 330-331, 40 EHRR 1030 : «[s]ur la base de l'ensemble des éléments en sa possession, la Cour estime que le gouvernement moldave, seul gouvernement légitime de la République de Moldova au regard du droit international, n'exerce pas d'autorité sur une partie de son territoire, à savoir celui se trouvant sous le contrôle effectif de la [République moldave de Transnistrie] [...] Toutefois, même en l'absence de contrôle effectif sur la région transnistrienne, la Moldova demeure tenue, en vertu de l'article 1 de la Convention, par l'obligation positive de prendre les mesures qui sont en son pouvoir et en conformité avec le droit international - qu'elles soient d'ordre diplomatique, économique, judiciaire ou autre - afin d'assurer dans le chef des requérants le respect des droits garantis par la Convention ». Pour en savoir plus, voir Gérard Cohen-Jonathan, « À propros des arrêts Assanidzé (8 avril 2004), Ilascu (8 juillet 2004) et Issa (16 novembre 2004) : Quelques observations sur les notions de juridiction et d'injonction » (2005) 64 Revue Trimestrielle des droits de l'homme 767; Grisel, supra note 72 aux pp 325 et suiv. 


\title{
III. L'obligation de poursuivre ou d'extrader les crimes contre l'humanité dans les projets d'articles
}

La facilitation sans cesse progressive des mouvements de personnes entre États entraine comme conséquence que les criminels peuvent franchir facilement les frontières internationales et échapper aux poursuites des autorités de l'État sur le territoire duquel ils ont commis des crimes. L'obligation de poursuivre ou d'extrader est l'un des nombreux outils de coopération en matière criminelle que les États élaborent pour contrer ce phénomène ${ }^{102}$. Elle est instituée pour soutenir la coopération internationale dans la lutte contre l'impunité en obligeant un État à extrader ou à poursuivre une personne trouvée sur son territoire si celle-ci est soupçonnée de certains crimes ${ }^{103}$. Il existe à ce jour plus de 60 traités multilatéraux qui contiennent une disposition aut dedere aut judicare ${ }^{104}$. Pourtant, en ce qui concerne les crimes internationaux fondamentaux, tels le génocide, les crimes de guerre autres que les infractions graves et la plupart des crimes contre l'humanité, il n'existe pas de traité comportant une telle obligation ${ }^{105}$. Une convention internationale sur la prévention et la répression des crimes contre l'humanité à laquelle les présents projets d'articles pourraient aboutir tend à élaguer cette lacune - essentiellement sous l'angle des crimes contre l'humanité et pour la communauté conventionnelle liée à cet instrument - en prescrivant une obligation aut dedere aut judicare en son article 9.

En effet, dans le cadre du texte provisoirement adopté, l'obligation de poursuivre les crimes contre l'humanité est d'abord affirmés au projet d'article 2 suivant lequel « les crimes contre l'humanité, qu'ils soient ou non commis en temps de conflit armé, sont des crimes au regard du droit international que les États s'engagent à prévenir et à punir ». C'est aux termes du projet d'article 9 intitulé «Aut dedere aut judicare » que l'obligation est mieux précisée. Pour le rappeler, ce projet d'article 9, dispose que :

\begin{abstract}
[1]'État sur le territoire sous la juridiction duquel l'auteur présumé de l'infraction se trouve soumet l'affaire à ses autorités compétentes pour l'exercice de l'action pénale, s'il n'extrade ou ne remet pas cette personne à un autre État ou à une juridiction pénale internationale compétente. Ces autorités prennent leur décision dans les mêmes conditions que pour toute autre infraction ayant un caractère grave en vertu du droit de cet État.
\end{abstract}

Le projet d'article 9 fait ainsi obligation à l'État sur le territoire sous la juridiction duquel l'auteur présumé d'avoir commis, tenté de commettre ou ordonné

\footnotetext{
102 Raphaël van Steenberghe, «The Obligation to Extradite or Prosecute. Clarifying its Nature » (2009) 9 Journal of International Criminal Justice 1089.

103 Shang Weiwei et Zhang Yueyao, «The Aut Dedere Aut Judicare Provision in the Proposed Convention on Crimes Against Humanity: Assessment from a Chinese perspective » dans Morten Bergsmo et Song Tianying, dir, On the Proposed Crimes Against Humanity Convention, Bruxelles, Torkel Opsahl Academic EPublisher, 2014 à la p 345.

104 Examen des conventions multilatérales qui pourraient présenter un intérêt pour les travaux de la Commission du droit international sur le sujet intitulé " L'obligation d'extrader ou de poursuivre (aut dedere aut judicare) », Doc off AG NU, (2010) Doc NU A/CN.4/630 (Étude du Secrétariat) au para 4 [Examen des conventions multilatérales].

105 Projets d'articles sur les crimes contre l'humanité, supra note 11.
} 
de commettre, sollicité, encouragé, apporté son aide, son concours ou toute autre forme d'assistance à la commission ou à la tentative de commission d'un crime contre l'humanité, de soumettre le cas à ses autorités compétentes pour l'exercice de l'action pénale ${ }^{106}$.

$\mathrm{Si}$, comme il a été noté tout au début, le régime conventionnel existant présent [ait] d'importantes lacunes quant à l'obligation d'extrader ou de poursuivre les crimes contre l'humanité et que les présents projets d'articles ont pour effet notamment de les combler, il importe, afin de comprendre le nouveau développement apporté au régime de l'obligation de poursuivre les crimes contre l'humanité, d'examiner sous l'angle de ce nouveau cadre censé répondre aux insuffisances décriées, le contenu et le sens de l'obligation prévue au projet d'article 9 sur les crimes contre l'humanité (A), et sa mise en œuvre (B).

\section{A. Le contenu et le sens de l'obligation aut dedere aut judicare prévue au projet d'article 9 tel qu'adopté à ce jour par la CDI}

L'obligation d'extrader ou de poursuivre, communément désignée par l'expression "aut dedere aut judicare », suggère l'existence d'une obligation générale composée de deux sous-obligations - celles d'extrader et de poursuivre entre lesquelles il convient de faire le choix. L'enseignement qui découle du projet d'article 9 instituant cette règle, en ce qui concerne les crimes contre l'humanité, est que celle-ci consiste en une obligation de poursuivre et non d'extrader ou de remise (1), entendue au sens d'une obligation de soumettre l'affaire aux autorités compétentes pour l'exercice de l'action pénale et indépendamment d'une demande d'extradition ou de remise (2).

\section{UNE OBLIGATION DE POURSUIVRE ET NON D’EXTRADER OU DE REMISE}

Le projet d'article 9 fait obligation à l'État de soumettre l'affaire à ses autorités compétentes pour l'exercice de l'action pénale, s'il n'extrade ou ne remet

106 Il en va de même, d'une part, de tout chef militaire ou une personne faisant effectivement fonction de chef militaire pour des crimes contre l'humanité commis par des forces placées sous son commandement et son contrôle effectifs, ou sous son autorité et son contrôle effectifs, selon le cas, lorsqu'il ou elle n'a pas exercé le contrôle qui convenait sur ces forces, dans les cas où ce chef militaire ou cette personne savait, ou, en raison des circonstances, aurait dû savoir, que ces forces commettaient ou allaient commettre ces crimes; et que ce chef militaire ou cette personne n'a pas pris toutes les mesures nécessaires et raisonnables qui étaient en son pouvoir pour en empêcher ou en réprimer l'exécution ou pour en référer aux autorités compétentes aux fins d'enquête et de poursuites. D'autre part, il en va également de même de tout supérieur hiérarchique pour des crimes contre l'humanité commis par des subordonnés placés sous son autorité et son contrôle effectifs, lorsqu'il n'a pas exercé le contrôle qui convenait sur ses subordonnés, dans les cas où, le supérieur hiérarchique savait que ses subordonnés commettaient ou allaient commettre ces crimes ou a délibérément négligé de tenir compte d'informations qui l'indiquaient clairement; que ces crimes étaient liés à des activités relevant de sa responsabilité et de son contrôle effectifs; et que le supérieur hiérarchique n'a pas pris toutes les mesures nécessaires et raisonnables qui étaient en son pouvoir pour en empêcher ou en réprimer l'exécution ou pour en référer aux autorités compétentes aux fins d'enquêtes et de poursuites (Ibid, arts $5,9)$. 
pas cette personne à un autre État ou à une juridiction pénale internationale compétente. Ainsi qu'il ressort des travaux préparatoires ${ }^{107}$, le projet d'article 9 s'inspire d'une disposition similaire contenue dans la Convention contre la torture de 1984, à savoir le premier paragraphe de l'article $7^{108}$ qui lui-même s'inspire de l'article 7 de la Convention de La Haye pour la répression de la capture illicite d'aéronef $\mathrm{s}^{109}$, signée à la Haye le 16 décembre 1970, ainsi que du paragraphe 1 de l'article 11 de la Convention internationale pour la protection de toutes les personnes contre les disparitions forcées adoptée le 20 décembre 2006 par l'Assemblée générale de l'ONU et entrée en vigueur le 23 décembre $2010^{110}$.

Sachant que la CIJ a déjà interprété la nature de l'obligation contenue au paragraphe 1 de l'article 7 de la Convention contre la torture, l'on peut dire que la règle «aut dedere aut judicare » prévue au projet d'article 9 ne met, à charge de l'État, qu'une obligation de poursuivre et non d'extrader ou de remise. La Cour ayant considéré, en effet, que le paragraphe 1 de l'article 7 impose en réalité à l'État concerné, l'obligation de soumettre l'affaire à ses autorités compétentes pour l'exercice de l'action pénale, indépendamment de l'existence, au préalable, d'une demande d'extradition à l'encontre du suspect ${ }^{111}$. La Cour a, au demeurant, déclaré que :

[1]e choix entre l'extradition et l'engagement des poursuites, en vertu de la convention contre la torture, ne revient pas à mettre les deux éléments de l'alternative sur le même plan [au sens où] l'extradition est une option offerte par la convention à l'État, alors que la poursuite est une obligation internationale, prévue par la convention, dont la violation engage la responsabilité de l'État pour fait illicite ${ }^{112}$.

Autrement dit, l'article $7 \S 1$ n'emporte aucune obligation d'extrader à défaut de poursuivre, l'obligation étant uniquement celle de poursuivre à défaut

107 Voir Sean D. Murphy, Rapporteur spécial, Deuxième rapport sur les crimes contre l'humanité, Doc off AG NU, 68 sess, Doc NU A/CN.4/690 (2016) aux paras 155-66 [Murphy, Deuxième rapport].

108 «L'État partie sur le territoire sous la juridiction duquel l'auteur présumé d'une infraction visée à l'article 4 est découvert, s'il n'extrade pas ce dernier, soumet l'affaire, dans les cas visés à l'article 5 , à ses autorités compétentes pour l'exercice de l'action pénale ». Convention contre la torture, supra note 59 , art 7.

109 « L'État contractant sur le territoire duquel l'auteur présumé de l'infraction est découvert, s'il n'extrade pas ce dernier, soumet l'affaire, sans aucune exception et que l'infraction ait ou non été commise sur son territoire, à ses autorités compétentes pour l'exercice de l'action pénale. Ces autorités prennent leur décision dans les mêmes conditions que pour toute infraction de droit commun de caractère grave conformément aux lois de cet État ». Convention de La Haye pour la répression de la capture illicite d'aéronefs, 16 décembre 1970, 860 RTNU 105 (entrée en vigueur : 14 octobre 1971), art 7 [Convention de La Haye de 1970].

110 «L'État partie sur le territoire sous la juridiction duquel l'auteur présumé d'un crime de disparition forcée est découvert, s'il n'extrade pas ce dernier, ou ne le remet pas à un autre État conformément à ses obligations internationales ou à une juridiction pénale internationale dont il a reconnu la compétence, soumet l'affaire à ses autorités compétentes pour l'exercice de l'action pénale ». Convention internationale pour la protection de toutes les personnes contre les disparitions forcées, 6 février 2007, 2716 RTNU 3 (entrée en vigueur : 23 décembre 2010) [Convention contre les disparitions forcées].

111 Affaire des questions concernant l'obligation de poursuivre ou d'extrader (Belgique c Sénégal), Arrêt du 20 juillet 2012, [2012] CIJ rec au para 94 [Belgique c Sénégal].

112 Ibid au para 95. 
d'extrader ${ }^{113}$. Toutefois, s'il appert de la lecture du projet d'article 9 que seule la poursuite constitue une obligation à charge de l'État alors que l'extradition ou la remise est une option, il n'en demeure pas moins vrai - aux termes des commentaires adoptés par la CDI à ce propos - que l'option de l'extradition du suspect ou de sa remise à une juridiction internationale compétente soit envisagée comme un moyen pour l'État qui décide de ne pas poursuivre le suspect trouvé sur son territoire, d'échapper au manquement découlant de cette absence de poursuite ${ }^{114}$. Il ressort de la première phrase du projet d'article 9 que l'État peut s'acquitter de l'obligation qui lui incombe soit en extradant le suspect vers un autre État, soit en le remettant à un tribunal pénal international compétent ${ }^{115}$. Cette troisième option est apparue avec la création de la Cour pénale internationale et d'autres tribunaux pénaux internationaux ${ }^{116}$. POUR L'EXERCICE DE L'ACTION PÉNALE

S'il est apparu plus convenable à la CDI d'intituler le projet d'article 9 : « Aut dedere aut judicare ${ }^{117}$ » et que le projet d'article 2 consacre un engagement des États de «punir » les crimes contre l'humanité, il est devenu très clair, au regard du développement précédent, que la seule obligation qui ressort des dispositions du projet d'article 9 est celle de « poursuivre à défaut d'extrader » et non de « poursuivre ou d'extrader ». Cela revient à dire que tant l'intitulé du projet d'article 9 (aut dedere aut judicare) que l'engagement de «punir» les crimes contre l'humanité prévu au projet d'article 2 sont, à première vue, trompeurs tant il n'existe dans le cadre des projets d'articles sur les crimes contre l'humanité, ni obligation d'en juger les auteurs, ni obligation de les punir.

113 Raphael van Steenberghe, «L'arrêt de la Cour internationale de justice dans l'Affaire Belgique contre Sénégal ou du principe aut dedere aut judicare » (2012) 45:2 Rev BDI 663 [Van Steenberghe].

114 Voir à ce sujet le commentaire $6 \mathrm{du}$ projet d'article 9. Projets d'articles sur les crimes contre l'humanité, supra note 11 à la p 286.

$115 \mathrm{Au}$ sujet de l'extradition ou de la remise, les commentaires annexés au projet d'article 9 relèvent que bien que l'«extradition» soit généralement associée au renvoi devant les autorités d'un État et la « remise » au renvoi devant un tribunal pénal international compétent, le projet d'article 9 a été libellé de manière à ne pas ainsi restreindre l'emploi de ces expressions. La Commission a préféré retenir une formulation couvrant toutes les situations sachant que la terminologie du droit pénal varie d'un État à l'autre et entre les instruments internationaux. Voir dans ce sens et pour illustration: Union européenne, Décision-cadre du Conseil du 13 juin 2002 relative au mandat d'arrêt européen et aux procédures de remise entre États membres, Journal officiel, n L 190, 18 juillet 2002 à la p 1. Aux termes de l'article premier de la Décision-cadre, «Le mandat d'arrêt européen est une décision judiciaire émise par un État membre en vue de l'arrestation et de [la remise] par un autre État membre d'une personne recherchée pour l'exercice de poursuites pénales ou pour l'exécution d'une peine ou d'une mesure de sûreté privatives de liberté » [Nos italiques].

116 Voir Rapport final de la CDI-aut dedere aut judicare, supra note 7 aux pp 161-62.

117 Suivant les commentaires adoptés et annexés au projet d'article 9, de crainte qu'une traduction littérale ne rende pas fidèlement le sens de l'expression « aut dedere aut judicare », la Commission a décidé de retenir celle-ci telle quelle dans le titre du projet d'article, d'autant qu'elle est couramment employée lorsqu'il est fait référence à l'obligation qu'elle recouvre. Voir à cet effet, Projets d'articles sur les crimes contre l'humanité, supra note 11 à la p 283. 
En limitant ainsi la formule à la seule obligation pour l'État de «soumettre l'affaire à ses autorités compétentes pour l'exercice de l'action pénale », le troisième commentaire adopté relativement au projet d'article 9 vient préciser qu'il suffit juste de saisir le ministère public, à charge pour celui-ci d'apprécier l'opportunité d'engager des poursuites ${ }^{118}$. L'on retiendra en particulier que si l'autorité compétente, c'est-à-dire le Ministère Public, estime qu'il n'existe pas d'indices suffisants de culpabilité, il n'y a pas lieu de juger ni de punir le suspect ${ }^{119}$. Il y a lieu de conclure aussi que la prétendue obligation de poursuivre n'existe pas à charge des États dès lors qu'une obligation de saisir le Ministère Public ne constitue pas une obligation de poursuivre ${ }^{120}$. Ainsi qu'il ressort des travaux préparatoires de la Convention pour la répression de la capture illicite d'aéronefs ${ }^{121}$, la formule consacre " [1']obligation de répression, [la possibilité] d'extradition, [l']obligation de transmission du dossier au ministère public et [la possibilité] de poursuites ${ }^{122} »$. Ce qui est clairement conforme à la volonté des États parties, la formule prévue dans les textes ayant en effet été choisie pour accommoder les nombreux États dans lesquels la décision de poursuivre relève du pouvoir discrétionnaire des autorités compétentes en la matière ${ }^{123}$.

Dès lors, en ce qui concerne le texte des projets d'articles sur les crimes contre l'humanité, les États ne font face qu'à la seule obligation de soumettre le suspect aux autorités nationales compétentes pour l'exercice de l'action pénale et, pour ces autorités, à se prononcer sur l'affaire de la même manière qu'elles le feraient au sujet d'affaires similaires en vertu du droit national. La deuxième phrase du projet d'article 9 est on ne peut plus claire à ce sujet. Elle prévoit que, lorsqu'un État exerce des poursuites pénales contre le suspect ou extrade ou remet celui-ci, ses « autorités prennent leur décision dans les mêmes conditions que pour toute autre infraction ayant un caractère grave en vertu du droit de cet État ». Cela revient à dire que ces dernières conservent donc leur pouvoir d'évaluer l'opportunité des poursuites étant entendu qu'elles gardent la maîtrise du déclenchement des poursuites, en fonction des preuves à leur disposition et des règles pertinentes de la procédure pénale, et sont ainsi libres de classer l'affaire sans suite sans que cette décision contrevienne à l'obligation imposée à l'État dont elles relèvent de «poursuivre » le suspect à défaut de l'extrader ou de le remettre à une juridiction internationale compétente. Il s'ensuit donc que les autorités compétentes étant maîtresses de l'opportunité des poursuites, un État, auquel il n'est fait que l'obligation de saisir ces dernières pour l'exercice de l'action pénale, peut se conformer aux obligations qui lui incombent au titre de ces projets d'articles sans que l'auteur présumé soit effectivement poursuivi, extradé ou remis.

118 Voir le commentaire 3 de l'article 9. Ibid à la p 284.

119 Ibid. Dans le même sens, Examen des conventions multilatérales, supra note 104 aux pp 74-75.

120 Dans le même sens, Delabie, supra note 8 à la p 398.

121 Voir article 7, Convention de La Haye de 1970, supra note 109.

122 Déclaration du Président, Gilbert Guillaume (France), Comité juridique de l'Organisation de l'aviation civile internationale, dix-septième session, Montréal, 9 février 11 mars 1970, Procès-verbaux et documents relatifs à la capture illicite d'aéronefs (Montréal, 1970), 30e séance (3 mars 1970) (document 8877-LC/161) au para 15.

123 G M E White, "The Hague Convention for the Suppression of Unlawful Seizure of Aircraft » (1971) The Review of the International Commission of Jurists à la p 42. 
Relativement à ce qui précède, il y a lieu de se questionner sur l'étendue d'un tel pouvoir d'appréciation de l'opportunité de poursuivre par les autorités compétentes. En ce qui concerne les crimes contre l'humanité, on ne peut se douter qu'ils portent gravement atteinte à la dignité humaine en tant que telle. La répression de ces crimes suggère que l'État amené à poursuivre l'auteur présumé est censé agir dans l'intérêt de la communauté des États, indépendamment de ses intérêts propres. Dans une telle hypothèse, comme le souligne Raphael van Steenberghe, il serait difficile d'admettre que les autorités compétentes pour l'exercice de l'action pénale soient autorisées à exercer un pouvoir discrétionnaire absolu dans leur décision d'engager les poursuites et qu'elles puissent, en particulier, invoquer des motifs de refus liés aux intérêts propres de l'État dont elles relèvent, tels que l'absence d'intérêt public pour cet État ${ }^{124}$.

Il est vrai qu'aucune disposition des projets d'article sous examen ne permet de fixer l'étendue de ce pouvoir d'appréciation de l'opportunité des poursuites. Par ailleurs, aucun élément susceptible d'infirmer ou de confirmer le rejet de l'exercice de ce pouvoir pour tout autre motif que l'insuffisance des charges contre l'auteur présumé ne peut être trouvé dans les commentaires tels qu'adoptés à ce jour par la CDI, au sujet desdits projets d'article. L'on peut néanmoins constater que la question a été antérieurement discutée dans le cadre des travaux de celle-ci relatifs au projet de code des crimes contre la paix et la sécurité de l'humanité ${ }^{125}$. En effet, au cours de l'élaboration de l'actuel article 9 portant sur l'obligation d'extrader ou de poursuivre, du projet de code des crimes contre la paix et la sécurité de l'humanité établi par la Commission en $1996^{126}$, on a d'abord pu soutenir que la règle ordinaire de l'opportunité des poursuites ne conviendrait pas pour les crimes visés dans le code, notamment les crimes contre l'humanité prévus à l'article 18 du projet de code ${ }^{127}$. Toutefois, à la suite de discussions il a été suggéré que l'écriture de l'article 9 reprenne la longue formule utilisée dans les traités multilatéraux prévoyant une telle obligation afin d'éviter qu'il soit interprété comme "a[yant] pour effet d'imposer automatiquement une obligation de poursuivre s'agissant de toute allégation de crime $^{128} »$ en l'absence d'extradition du suspect. Cette proposition s'expliquait en

124 Van Steenberghe, supra note 113 aux pp 683-84.

125 Nous pouvons toutefois souligner que le deuxième rapport sur les crimes contre l'humanité présenté par Sean Murphy à la Soixante-huitième session (Murphy, Deuxième rapport, supra note 107 aux pp 80-81), reprend quelques extraits des commentaires de l'article 9 du projet de code des crimes contre la paix et la sécurité de l'humanité de 1966, mais aucun autre commentaire n'en a été dans le texte des projets d'article tel qu'adopté à ce jour adopté afin de savoir si, en ce qui concerne le projet d'article 9 et plus particulièrement, l'opportunité des poursuites, les rédacteurs ont entendu s'en inspirer ou pas.

126 Annuaire de la Commission du droit international 1996, vol II (deuxième partie), chap II, sect D, Doc off AG NU, 48 sess, Doc NU A/CN.4/SER.A/1996/Add.1, art 9 [Annuaire de la CDI].

127 Voir l'explication de M Calero Rodrigues, Président du Comité de rédaction, Ibid, vol. I à la p 53 au para 36 : «Le Comité a en outre remplacé le membre de phrase « extrade ce dernier ou soumet l'affaire à ses autorités compétentes pour l'exercice de l'action pénale » par " extrade ou poursuit ce dernier », car, dans le premier libellé, on admettait la notion d'opportunité des poursuites, notion mal adaptée au projet de code $»$.

128 Voir notamment le résumé des déclarations de M Crawford, Ibid au para 37 : «M. Crawford est hostile à la modification apportée par le Comité de rédaction à la session en cours, dans la mesure où elle a pour effet d'imposer automatiquement une obligation de poursuivre s'agissant de toute allégation de crime, ce qui est contraire aux principes normaux de l'entraide judiciaire internationale. Il convient que, 
l'occurrence, comme le relève à juste titre Van Steenberghe, par le souci de permettre aux autorités chargées des poursuites de décider de ne pas engager les poursuites dans l'hypothèse où les preuves ne seraient pas suffisantes ${ }^{129}$. Si la proposition n'a toutefois pas été retenue, l'actuel article 9 ayant gardé le même libellé retenu par le comité de rédaction, suivant lequel «[s]ans préjudice de la compétence d'une cour criminelle internationale, l'État partie sur le territoire duquel l'auteur présumé d'un crime visé aux articles 17, 18, 19 ou 20 est découvert extrade ou poursuit ce dernier », le commentaire y annexé prend soin de préciser d'une part que « [1]'État de détention [est] dans l'obligation de poursuivre l'auteur présumé d'un crime sur son territoire lorsqu'il y [a] suffisamment de preuves pour ce faire au regard de son droit interne, à moins qu'il ne décide de faire droit à une demande d'extradition ${ }^{130} \gg$ et, d'autre part, que «la liberté d'appréciation reconnue dans certains systèmes juridiques quant à l'opportunité des poursuites, grâce à laquelle l'auteur présumé d'un crime peut se voir accorder l'immunité en échange d'un témoignage ou d'un concours prêté aux poursuites exercées contre un autre individu dont le comportement criminel est considéré comme plus grave, est exclue pour les crimes visés par le présent code ${ }^{131} »$. La Commission explique cette prise de position par le fait que

les crimes internationaux constituent les crimes les plus graves qui intéressent la communauté internationale dans son ensemble [et] [c]'est tout particulièrement le cas des crimes contre la paix et la sécurité de l'humanité visés par le code. [Dès lors,] il serait contraire aux intérêts de l'ensemble de la communauté internationale de permettre à un État de conférer l'immunité à un individu qui serait responsable d'un crime international $(\ldots)^{132}$

Des commentaires ci-haut, il appert que si l'on ne peut affirmer une interdiction générale, pour les autorités compétentes chargées des poursuites pénales, d'exercer leur pouvoir discrétionnaire d'appréciation de l'opportunité des poursuites à

dans le contexte des crimes visés dans le projet de code, la règle ordinaire de l'opportunité des poursuites serait mal venue. Il peut cependant exister de nombreuses raisons de ne pas poursuivre une personne, qui n'ont rien à voir avec une décision de ne pas exercer à son encontre des poursuites au fond : par exemple, il se pourrait qu'il n'existe aucun élément prouvant que la personne en question a effectivement commis un crime ».

129 Van Steenberghe, supra note 103 aux pp 1109-10: "The main concern was to preserve the discretionary power of the relevant authority not to have to initiate prosecution in cases lacking sufficient evidence. Such a situation is one of the main discretionary reasons for not prosecuting a case in domestic legal systems recognizing prosecutorial discretion. This reason is common to many national systems, including those which do not admit such discretion. In those systems, it is qualified as a legal rather than a discretionary circumstance imposing on the relevant authorities an obligation to dismiss the case. It therefore seems that such a reason, although being part of the discretionary power of prosecutorial authorities in some national systems, must be taken into account when prosecuting a suspect, even with respect to core international crimes. It is this solution that has been finally adopted by the ILC. While the wording 'obligation to extradite or prosecute' was retained in the final version of the Draft Code, the commentary to the Draft Code mentions that the custodial state [will] have an obligation to prosecute an alleged offender in its territory when there [is] sufficient evidence for doing so as a matter of national law unless it decide[s] to grant a request received for extradition $\gg$.

130 Voir paragraphe $4 \mathrm{du}$ commentaire de l'article 9. Projets d'articles sur les crimes contre l'humanité, supra note 11 à la $\mathrm{p} 32$.

131 Ibid.

132 Ibid. 
l'égard des crimes contre l'humanité, certaines limites à l'exercice de ce pouvoir sont, au demeurant, envisageables. Elles procèderaient nettement du fait que la répression de ces crimes - qualifiés au projet d'article 2 des crimes de droit international que les États s'engagent notamment à punir - vise la protection d'intérêts propres à la communauté internationale dans son ensemble, transcendant donc ceux des États. Il serait absurde que des motifs de refus de poursuivre autre que l'insuffisance de charges, à l'instar de celui découlant de l'absence d'intérêt national, soient admis.

\section{UNE OBLIGATION DE POURSUIVRE INDÉPENDANTE D’UNE DEMANDE D’EXTRADITION OU DE REMISE}

Une disposition similaire au projet d'article 9 est contenue au premier paragraphe de l'article 7 de la Convention contre la torture de 1984, qui lui-même s'inspire de l'article 7 de la Convention pour la répression de la capture illicite d'aéronefs. Ce projet d'article s'inspire aussi du paragraphe 1 de l'article 11 de la Convention internationale pour la protection de toutes les personnes contre les disparitions forcées. Construit sur le modèle de ces conventions, l'interprétation de l'obligation posée au projet d'article 9 n'est pas sans poser des difficultés dans la mesure évidente où les termes dans lesquels ces conventions prévoient l'obligation de poursuivre à défaut d'extrader ne permettent pas, à première vue, d'établir si l'application de cette obligation dépend ou non d'une demande préalable d'extradition. Il convient de noter que les travaux préparatoires de la Convention pour la répression de la capture illicite d'aéronefs attestent que faute de s'être accordés sur ce sujet, les États ont volontairement choisi de garder une ambiguïté dans la Convention $^{133}$ qui est nettement évoquée dans le document du Secrétariat général des Nations, rédigé en vue d'assister la CDI dans ses travaux relatifs à l'obligation d'extrader ou de poursuivre, dans lequel on peut en effet lire :

les termes de la disposition pertinente contenue dans la Convention pour la répression de la capture illicite d'aéronefs (la Convention de La Haye de 1970) apparaissent ambigus à cet égard : en effet, [...] ils prévoient que [l'] État contractant sur le territoire duquel l'auteur présumé de l'infraction est découvert, s'il n'extrade pas ce dernier, soumet l'affaire [...] à ses autorités compétentes pour l'exercice de l'action pénale. [...] Même en tenant compte du contexte dans lequel il s'inscrit et des travaux préparatoires à la Convention, le texte de cette disposition ne résout pas sans la moindre ambiguité la question de savoir si l'obligation de poursuivre naît ipso facto, ou seulement à partir du moment où une demande d'extradition a été présentée et rejetée. En outre, les vues exprimées dans la doctrine sur les dispositions de ce type ne tranchent pas la question de façon indiscutable ${ }^{134}$.

Dans l'arrêt qu'elle a rendu dans l'Affaire des questions relatives à l'obligation de poursuivre ou d'extrader (Belgique c Sénégal), la Cour internationale

133 Van Steenberghe, supra note 113 à la p 691.

134 Voir Examen des conventions multilatérales, supra note 104 aux paras 130, 315-16. 
de justice s'est intéressée à la formule de La Haye au regard de l'article 7 de la Convention contre la torture. Elle a en effet considéré que «le paragraphe 1 de l'article 7 impos[ait] à l'État concerné l'obligation de soumettre l'affaire à ses autorités compétentes pour l'exercice de l'action pénale, indépendamment de l'existence, au préalable, d'une demande d'extradition à l'encontre du suspect», ajoutant que « [c'était] pour cela que le paragraphe 2 de l'article 6 oblige[ait] l'État à procéder immédiatement à une enquête préliminaire, aussitôt que le suspect se trouv[ait] sur son territoire ${ }^{135} »$. La Cour a même tenu à noter que l'extradition n'était qu'une option ${ }^{136}$. L'ambiguité susévoquée pourrait à cet effet être dissipée.

Ceci dit, le projet d'article 9 ne fait donc pas dépendre l'application de l'obligation de poursuivre qu'il contient à une demande d'extradition ou de remise. Ce qui compte est que le suspect soit immédiatement poursuivi une fois qu'il est identifié sur le territoire. L'option de l'extradition vers un autre État que celui sur le territoire duquel l'auteur présumé des crimes contre de l'humanité est découvert ou celle de la remise du suspect à une cour pénale internationale compétente n'est donc pas à considérer en premier lieu. L'accent est ainsi mis sur les poursuites par l'État de détention, alors que l'option de l'extradition ou de la remise se présente seulement comme une voie alternative aux poursuites ${ }^{137}$. Le fait que la CDI ait intitulé le projet d'article 9 par la formule usuelle "aut dedere aut judicare », plaçant ainsi l'option d'extradition en premier lieu ne semble pas adaptée pour exprimer l'obligation contenue dans ce projet d'article. En d'autres termes, cette obligation ne signifie pas nécessairement que des poursuites seront engagées, et encore moins que l'auteur présumé sera puni tel qu'il a été précédemment relevé. C'est pourquoi il aurait été préférable d'intituler le projet d'article 9 par la formule «aut dedere aut prosequi » ou plus rigoureusement, par la formule " prosequi vel dedere $e^{138} »$. Le commentaire du projet d'article révèle que le choix opéré par la CDI de l'expression « aut dedere aut judicare » est justifié par la seule « crainte qu'une traduction littérale n['en] rende pas fidèlement le sens ${ }^{139} »$. Elle a préféré utiliser, selon ses propres termes, «l'expression telle qu'elle est couramment employée lorsqu'il est fait référence à l'obligation qu'elle recouvre ${ }^{140} »$. L'argument ne semble pas, pour autant, solide dès lors que l'expression contemporaine "aut dedere aut judicare » signifie littéralement «soit extrader (ou livrer), soit juger » et ne rend pas aussi fidèlement le même sens que la nature de l'obligation prévue au projet d'article 9 permet de lui donner. Les travaux antérieurs de la CDI révèlent à ce sujet que dans le passé, certains membres de la

135 Belgique c Ségénal, supra note 111 au para 94.

136 Ibid au para 95.

137 Il en serait ainsi notamment lorsque la décision de ne pas poursuivre a été prise sur des motifs illicites incompatibles avec l'indépendance du procureur ou si la procédure judiciaire a été engagée dans le but de protéger le suspect de sa responsabilité pénale.

138 Voir Gilbert Guillaume, « Terrorisme et droit international» (1989) 215, t 3, Rec des Cours à la p 287 : on peut lire à la page 371 que « [d]ès lors la véritable option qui s'ouvre aux États est nécessairement aut dedere, aut prosequi ». Voir également Marc Henzelin, Le Principe de l'universalité en droit Pénal International: Droit et Obligation pour les États de Poursuivre et Juger selon le Principe de l'universalité, Bruxelles, Bruylant, 2000.

139 Voir le paragraphe $1 \mathrm{du}$ commentaire de l'article 9. Projets d'articles sur les crimes contre l'humanité, supra note 11 à la $\mathrm{p} 287$.

140 Ibid. 
Commission, dont le Rapporteur spécial M Zdzislaw Galicki, avait douté de l'opportunité d'employer la formule latine "aut dedere aut judicare », surtout en ce qui concerne le mot «judicare », qui n'est pas véritablement l'équivalent de «poursuivre ». Mais le rapporteur spécial avait cependant considéré qu'il était à ce stade prématuré de se concentrer sur le sens précis à donner aux termes utilisés, cela devant être fait dans un futur projet d'article consacré aux "définitions ${ }^{141}$ ». Ce qui n'a pas été fait à ce jour.

\section{B. La mise en œuvre de l'obligation de poursuivre}

Dans le contexte des présents projets d'articles sur les crimes contre l'humanité, l'obligation de poursuivre prévue au projet d'article 9 est normalement mise en œuvre après que l'État s'est acquitté des autres obligations prévues dans les articles précédents, qui lui imposent d'adopter une législation adéquate pour incriminer les crimes contre l'humanité, de conférer à ses tribunaux une compétence universelle en la matière et d'effectuer une enquête pour établir les faits. Pour reprendre le raisonnement de la Cour internationale de justice dans l'Affaire des Questions concernant l'obligation de poursuivre ou d'extrader (Belgique c Sénégal), « ces obligations, dans leur ensemble, peuvent être considérées comme des éléments d'un même dispositif conventionnel visant à éviter que les suspects ne puissent échapper à la mise en jeu, s'il y a lieu, de leur responsabilité pénale ${ }^{142}$ ». Certes, le raisonnement de la Cour se rapporte spécifiquement à la mise en œuvre de l'obligation de poursuivre dans le cadre de la Convention contre la torture. Mais, les dispositions de celle-ci relatives à l'obligation de poursuivre à défaut d'extrader suivent le modèle de la «formule de La Haye » dont s'inspire les présents projets d'articles sur les crimes contre l'humanité. Il va sans dire que la décision de la Cour peut aussi contribuer à préciser le sens du régime de l'obligation de poursuivre à défaut d'extrader contenu dans lesdits projets d'articles ainsi que les éléments fondamentaux à inclure dans les législations nationales pour une exécution effective de l'obligation de poursuivre. Cela suppose en l'espèce que l'État prenne les mesures nécessaires au plan national pour incriminer les infractions concernées (1), établir sa compétence à l'égard des crimes contre l'humanité et de la personne présente sur son territoire (2), procéder à une enquête ou mener une enquête préliminaire (3) et enfin, exécuter l'obligation de poursuivre proprement dite en soumettant l'affaire aux autorités compétentes pour l'exercice de l'action pénale.

\section{L'OBLIGATION D'INCRIMINATION EN DROIT INTERNE}

L'expression «crimes contre l'humanité » étant définie aux paragraphes 1 à 3 du projet d'article 3, l'obligation d'incrimination en droit interne énoncée au projet d'article 5 suppose que les crimes ainsi définis soient érigés en infractions au regard

141 Voir Zdzislaw Galicki, Rapporteur spécial, Troisième rapport sur l'obligation d'extrader ou de poursuivre (aut dedere aut judicare), Doc off $\mathrm{AG} \mathrm{NU}, 60^{\mathrm{e}}$ sess, Doc NU A/CN.4/603 (2008) aux paras 36-37.

142 Belgique c Sénégal, supra note 111 au para 91. 
des lois pénales de l'État. En effet, le projet d'article 5 fait état de diverses mesures « nécessaires » et indispensables à la bonne application des projets d'articles suivants relatifs à l'établissement et à l'exercice de la compétence à l'égard des auteurs présumés des crimes contre l'humanité, que tout État doit prendre dans le cadre de son droit interne pour que les crimes contre l'humanité constituent des infractions au regard de son droit pénal. Si le paragraphe 1 du projet d'article 5 énonce une obligation générale de criminaliser les crimes contre l'humanité en droit interne ${ }^{143}$, les paragraphes suivants énoncent des mesures spécifiques que l'État est amené à prendre quant à son effectivité. Il en est ainsi de la prise par l'État des mesures nécessaires pour que certains actes par lesquels des individus sont susceptibles de participer à des crimes contre l'humanité soient érigés en infractions au regard de son droit pénal : le fait de commettre un crime contre l'humanité, le fait de tenter de commettre un tel crime, le fait d'ordonner, solliciter, encourager, apporter son aide, son concours ou toute autre forme d'assistance à la commission ou à la tentative de commission d'un tel crime ${ }^{144}$. L'obligation de criminalisation en droit interne pose encore que l'État prenne des mesures législatives visant la question de la responsabilité du commandement ou du supérieur hiérarchique ${ }^{145}$. Les mesures législatives à prendre par l'État devront exclure toute possibilité d'invoquer comme moyen de défense l'ordre d'un supérieur ${ }^{146}$ ou une éventuelle prescription ${ }^{147}$, et prévoir des peines appropriées tenant compte de la gravité de ces crimes ${ }^{148}$.

Les paragraphes 1 à 6 du projet d'article 5 concernent la responsabilité pénale des personnes physiques quoique le terme «physique » n'y soit pas employé. Le commentaire relève, à ce sujet, que c'est dans un souci de cohérence avec les traités relatifs à la criminalité ${ }^{149}$. Le paragraphe 7 , quant à lui, traite de la responsabilité des «personnes morales » à l'égard des crimes contre l'humanité. Aux termes de ce dernier paragraphe, tout État devra prendre, sous réserve des dispositions de son droit interne, les mesures qui s'imposent, afin d'établir la responsabilité des personnes morales pour les infractions visées dans le projet d'article 5 qui, selon les principes juridiques de l'État, peut être pénale, civile ou administrative ${ }^{150}$.

143 Projet d'article 5 au para 1 : «Tout État prend les mesures nécessaires pour que les crimes contre l'humanité constituent des infractions au regard de son droit pénal » (Projets d'articles sur les crimes contre l'humanité, supra note 11, art 5(1)).

144 Ibid au para 2 : «Tout État prend les mesures nécessaires pour que les actes suivants constituent des infractions au regard de son droit pénal : a) Le fait de commettre un crime contre l'humanité; b) Le fait de tenter de commettre un tel crime; et c) Le fait d'ordonner, solliciter, encourager, apporter son aide, son concours ou toute autre forme d'assistance à la commission ou à la tentative de commission d'un tel crime $\gg$.

145 Projets d'articles sur les crimes contre l'humanité, supra note 11.

146 Ibid au para $4:$ «Tout État prend les mesures nécessaires pour que, au regard de son droit pénal, le fait qu'une infraction visée dans le présent projet d'article a été commise sur les ordres d'un gouvernement ou d'un supérieur, militaire ou civil, ne constitue pas un motif d'exonération de la responsabilité pénale d'un subordonné »).

147 Ibid au para 5 : « Tout État prend les mesures nécessaires pour que, au regard de son droit pénal, les infractions visées dans le présent projet d'article ne se prescrivent pas ».

148 Ibid au para 6: «Tout État prend les mesures nécessaires pour que, au regard de son droit pénal, les infractions visées dans le présent projet d'article soient passibles de peines appropriées qui prennent en compte leur gravité ».

149 Ibid, commentaire 36.

150 Ibid, art 5 au para 7. 


\section{2. ÉTABLISSEMENT DE LA COMPÉTENCE NÉCESSAIRE}

Le projet d'article 6 dispose que chaque État doit établir sa compétence aux fins de connaître des infractions visées au projet d'article 5 dans certains cas - par exemple lorsque le crime se produit sur un territoire sous sa juridiction - a été commis par l'un de ses ressortissants ou lorsque l'auteur de l'infraction se trouve sur un territoire sous sa juridiction. L'établissement de la compétence est « en toute logique, une étape préalable » à la mise en œuvre d'une obligation de poursuivre un suspect présent sur le territoire d'un État ${ }^{151}$. Ainsi que le relève le deuxième commentaire apporté à cet article 6 , d'une manière générale, les instruments internationaux visent à encourager les États à établir en droit interne un éventail assez large de chefs de compétence pour saisir les crimes les plus graves de portée internationale, et priver leurs auteurs de tout sanctuaire ${ }^{152}$. L'on peut à cet effet rappeler que déjà en 1996, il avait été posé, aux termes de l'article 8 du projet de code des crimes contre la paix et la sécurité de l'humanité, que «chaque État partie prend les mesures nécessaires pour établir sa compétence aux fins de connaître des crimes » visés dans le projet de code à l'exception de celui d'agression, bien entendu - «quels que soient le lieu ou l'auteur de ces crimes ${ }^{153}$. Une telle compétence aussi étendue s'imposait d'autant que la «Commission [avait] estimé que l'application effective [dudit] code exigeait, en matière de compétence, une approche conjuguant la plus large compétence des juridictions nationales avec la compétence possible d'une cour criminelle internationale $^{154} »$. Elle a ajouté que l'« obligation d'extrader ou de poursuivre » définie à l'article 9 du projet de Code des crimes de 1996 est étroitement liée à l'établissement de la «compétence » en vertu de l'article 8 du projet du même code, qui impose à chaque État partie de prendre les mesures nécessaires pour établir sa compétence aux fins de connaître de crimes visés, quels que soient le lieu ou l'auteur de ces crimes. Le commentaire de l'article 8 élaboré par la Commission à cet effet, indique clairement que c'est la compétence universelle qui est ainsi envisagée ${ }^{155}$.

Dans son opinion individuelle jointe à l'arrêt rendu en l'Affaire du Mandat d'arrêt, le juge Guillaume a fait une observation au sujet du « système » institué par les conventions prévoyant une obligation «aut dedere aut judicare ». Pour lui, " [1]orsque l'auteur de l'une des infractions couvertes par ces conventions est trouvé sur le territoire d'un État, ce dernier est dans l'obligation de l'arrêter, puis de l'extrader ou de le poursuivre. Il doit avoir au préalable conféré compétence à ses

151 Voir Rapport du Groupe d'experts techniques ad hoc Union africaine-Union européenne (UA-UE) sur le principe de compétence universelle (8672/1/09/Rev.1), annexe au para 11. Dans l'Affaire des questions concernant l'obligation de poursuivre ou d'extrader (Belgique c Sénégal), la Cour internationale de justice relève que la mise en œuvre par l'État de son obligation d'établir la compétence universelle de ses juridictions pour connaître du crime de torture est une condition nécessaire pour pouvoir procéder à une enquête préliminaire et soumettre l'affaire à ses autorités compétentes pour l'exercice de l'action pénale (Belgique c Sénégal, supra note 112 au para 74). Dans la même idée, voir le Rapport final de la CDI - aut detere aut judicare, supra note 7 à la p 155 .

152 Projets d'articles sur les crimes contre l'humanité, supra note 11, commentaire 2 à la p 276.

153 Annuaire de la CDI, supra note 126, art 8.

154 Projets d'articles sur les crimes contre l'humanité, supra note 11, commentaire 5.

155 Rapport de la Commission du Droit international sur son travail de la $48^{e}$ session, Doc off AG NU, $48^{\mathrm{e}}$ sess, supp n¹0, Doc NU. A/51/10 (1996) au para 7. 
tribunaux pour juger l'intéressé en l'absence d'extradition. De la sorte est assurée une répression universelle des infractions en cause, leurs auteurs ne pouvant trouver refuge sur le territoire d'aucun État ${ }^{156} »$.

L'on peut constater que dans le cadre des crimes contre l'humanité, le projet d'article 6 fait effectivement obligation à chaque État d'établir sa compétence aux fins de connaitre des infractions visées au projet d'article 5 lorsque le crime se produit sur un territoire sous sa juridiction, a été commis par l'un de ses ressortissants ou lorsque l'auteur de l'infraction se trouve sur un territoire sous sa juridiction, et dans le cas où il ne l'extrade ou ne le remet pas ${ }^{157}$. L'établissement des différents types de compétence nationale prévus par le projet d'article 6 est donc important pour appuyer l'obligation de poursuivre, telle qu'énoncée au projet d'article 9 sur les crimes contre l'humanité.

Ainsi qu'il ressort des commentaires ${ }^{158}$, le paragraphe 1 a) du projet d'article 6 prescrit à l'État sur le territoire duquel l'infraction a été commise d'établir sa compétence à l'égard de celle-ci, compétence dite «territoriale». Au lieu de mentionner uniquement le « territoire » de l'État, la CDI a jugé approprié de viser tout territoire «sous sa juridiction», de façon à englober le territoire de jure de l'État, ainsi que celui placé sous sa juridiction ou son contrôle de facto, le territoire occupé bien entendu. En outre, la compétence territoriale s'étend souvent aux infractions commises à bord de navires ou d'aéronefs immatriculés dans l'État; de fait, les États qui répriment les crimes contre l'humanité établissent d'ordinaire leur compétence à l'égard de tous actes commis à bord de tels navires ou aéronefs.

Le paragraphe $1 \mathrm{~b}$ ), prescrit à l'État dont l'auteur présumé de l'infraction est ressortissant d'établir sa compétence aux fins de connaître de cette infraction, cette compétence fondée sur la nationalité de l'auteur de l'infraction étant aussi appelée « compétence personnelle active ». En outre, selon le même paragraphe, l'État peut, s'il le souhaite, établir sa compétence lorsque l'auteur est « un apatride qui réside habituellement sur son territoire ».

Le paragraphe $1 \mathrm{c}$ ), vise la compétence de l'État dont la victime de l'infraction est ressortissante, une compétence aussi appelée «compétence personnelle passive ». Étant donné que de nombreux États préfèrent ne pas exercer cette compétence, elle est facultative; un État peut se déclarer compétent «s'il le juge approprié », mais il n'est pas tenu de le faire.

En son paragraphe 2, le projet d'article 6 envisage l'hypothèse où, d'autres titres de compétence ne trouvant pas application, l'auteur présumé de l'infraction « se trouve » cependant sur un territoire relevant de la juridiction de l'État et où celui-ci n'extrade ou ne remet pas cette personne conformément au présent projet d'articles. En pareil cas, même si le crime n'a pas été commis sur son territoire, et ni l'auteur

\footnotetext{
156 Affaire du Mandat d'arrêt (République démocratique du Congo c Belgique), arrêt du 11 avril 2000, [2002] CIJ, rec à la p 3, Opinion individuelle de M Guillaume, au para 9.

157 Voir l'article 6 du projet : «Établissement de la compétence nationale». Projets d'articles sur les crimes contre l'humanité, supra note 11 , art 6.

158 Ibid, commentaires 6-10 aux pp 277-278.
} 
présumé ni les victimes ne sont ses ressortissants, l'État est néanmoins tenu d'établir sa compétence, dès lors que l'auteur présumé se trouve sur un territoire sous sa juridiction. L'intérêt de cette obligation est d'empêcher l'auteur présumé de l'infraction de tenter de trouver refuge dans un État sans lien quelconque avec l'infraction. Il se comprend sans peine qu'aux fins du présent paragraphe, dans l'hypothèse où le crime a été commis à l'étranger et en l'absence de lien particulier avec l'État du for, l'obligation de poursuivre correspondrait nécessairement à l'exercice de la compétence universelle que les États doivent instituer dans la législation interne pour une répression efficiente des crimes contre l'humanité ${ }^{159}$.

Enfin, le paragraphe 3 indique clairement que si tout État a l'obligation d'établir certains types de compétence, cela n'exclut aucun autre chef de compétence prévu par le droit interne de l'État concerné, dès lors que pour sauvegarder le droit de tout État partie d'aller au-delà des prescriptions conventionnelles en établissant sa compétence nationale, et sans préjudice des règles de droit international applicables, les traités répressifs laissent généralement ouverte la possibilité qu'un État partie ait établi d'autres chefs de compétence juridictionnelle pour amener l'auteur présumé de telle ou telle infraction à en répondre ${ }^{160}$. Les juges Higgins, Kooijmans et Buergenthal l'ont fait observer lorsque dans leur opinion individuelle commune en l'Affaire $d u$ Mandat d'arrêt, citant notamment une disposition analogue de la Convention contre la torture, ils ont déclaré

[qu'ils ne repoussaient pas] l'idée que la lutte contre l'impunité est 'transférée' aux traités et tribunaux internationaux, les tribunaux nationaux n'ayant pas compétence en la matière. On a pris grand soin lorsqu'on a formulé les dispositions conventionnelles pertinentes, de ne pas exclure d'autres chefs de compétence, laquelle peut être exercée sur une base volontaire ${ }^{161}$.

Sur le plan des retards dans l'adoption de la législation, les projets d'articles ne prescrivent pas un délai précis. Toutefois, l'adoption de la législation instituant une compétence nationale à l'égard des crimes contre l'humanité doit se faire sans retard pour ne pas affecter l'exécution par l'État de son obligation de poursuivre. Mieux,

159 Selon un auteur, le principe aut dedere aut judicare recouvre partiellement la compétence universelle lorsqu'un État n'a pas d'autre lien avec le crime allégué ou le suspect que la simple présence de la personne sur son territoire (Mitsue Inazumi, Universal Jurisdiction in Modern International Law: Expansion of National Jurisdiction for Prosecuting Serious Crimes under International Law, Antwerp, Intersentia, 2005 à la p 122).

160 Voir Comité spécial sur l'élaboration d'une convention contre la criminalité transnationale organisée, Projet de convention des Nations Unies contre la criminalité transnationale organisée : texte révisé, Doc off AG NU, (1999) Doc NU A/AC.254/4/Rev.4, note 102 à la p 20]; voir également Conseil de l'Europe, Rapport explicatif de la Convention pénale sur la Corruption, Série des traités européens, $n^{\circ} 173$ au para 83 ( « La compétence repose traditionnellement sur la territorialité ou la nationalité. Or, en matière de corruption, ces principes ne sont pas toujours suffisants pour qu'un État puisse exercer sa compétence, par exemple lorsque l'infraction est commise hors du territoire d'une Partie contractante ou n'implique pas ses ressortissants, tout en affectant ses intérêts (par exemple, la sécurité nationale). Le paragraphe 4 de cet article autorise les Parties à établir, conformément à leur législation nationale, d'autres types de compétence $")$.

161 Affaire du génocide bosniaque, supra note 56, Opinion individuelle commune de Mme. Higgins, M Kooijmans et M Buergenthal au para 51. 
l'obligation doit être mise en œuvre par l'État sitôt qu'il est lié par la convention (à entendre par ici la future convention sur les crimes contre l'humanité que les présents projets d'articles sont appelés à devenir). Cela est rappelé par la CIJ à propos de la torture lorsqu'elle affirme dans l'Affaire des questions concernant l'obligation de poursuivre ou d'extrader (Belgique c Sénégal) que le fait que la législation nécessaire pour l'exercice de l'action pénale ait été adoptée tardivement affecte l'exécution par l'État partie de son obligation de procéder à une enquête préliminaire et de soumettre l'affaire à ses autorités compétentes pour l'exercice de l'action pénale ${ }^{162}$.

\section{OBLIGATIONS DE PROCÉDER À UNE ENQUÊTE IMMÉDIATE ET IMPARTIALE ET À UNE ENQUÊTE PRÉLIMINAIRE APRÈS PLACEMENT EN DÉTENTION DE L'AUTEUR PRÉSUMÉ}

En règle générale, l'obligation de mener une enquête doit être interprétée à la lumière de l'objet et du but de la convention applicable, qui est d'accroître l'efficacité de la lutte contre l'impunité ${ }^{163}$. L'obligation vise à corroborer ou non les soupçons qui pèsent sur la personne concernée ${ }^{164}$. Dans le cadre des présents projets d'articles sur les crimes contre l'humanité, deux obligations distinctes d'enquêter mais qui concourent aux mêmes fins, à savoir, la lutte contre l'impunité des crimes contre l'humanité semblent se dégager de l'analyse. En effet, d'une part, le projet d'article 7 pose une obligation générale d'enquêter pour tout État qui a des motifs raisonnables de croire que des actes constitutifs de crimes contre l'humanité ont été commis ou sont en voie d'être commis sur un territoire relevant de sa juridiction de veiller à ce que ses autorités compétentes procèdent à une enquête immédiate et impartiale ${ }^{165}$. Cette enquête permet à l'État non seulement d'identifier les auteurs présumés et de déterminer le lieu où ils se trouvent, mais aussi de prévenir la perpétuation ou la répétition des crimes en décelant leur l'origine. Une obligation analogue figure dans certains autres traités répressifs ${ }^{166}$. Ainsi en est-il de l'article 12 de la Convention contre la torture $^{167}$. Le Comité contre la torture a conclu à des violations de cet article lorsque l'enquête de l'État n'a pas été «immédiate et impartiale ${ }^{168}$ ». Si l'exigence de

162 Belgique c Sénégal, supra note 111 aux paras 76-77.

163 Ibid au para 86.

164 Ibid au para 83.

165 Projets d'articles sur les crimes contre l'humanité, supra note 11, art 7 : Enquête « Tout État veille à ce que les autorités compétentes procèdent immédiatement à une enquête impartiale chaque fois qu'il y a des motifs raisonnables de croire que des actes constitutifs de crimes contre l'humanité ont été commis ou sont en voie d'être commis sur tout territoire sous sa juridiction »).

166 Voir par exemple Convention interaméricaine pour la prévention et la répression de la torture, 9 décembre 1985, OAS TS NO 67 (entrée en vigueur : 28 février 1987), art 8; Convention contre les disparitions forcées, supra note 110, art 12(2); Convention du Conseil de l'Europe sur la prévention et la lutte contre la violence à l'égard des femmes et la violence domestique (Istanbul, 11 mai 2011), Conseil de l'Europe, Série des traités européens, $\mathrm{n}^{\circ} 210$, art 55(1).

167 «Tout État partie veille à ce que les autorités compétentes procèdent immédiatement à une enquête impartiale chaque fois qu'il y a des motifs raisonnables de croire qu'un acte de torture a été commis sur tout territoire sous sa juridiction » Convention contre la torture, supra note 59, art 12.

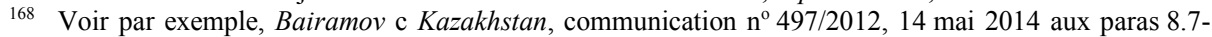
8.8, dans le Rapport du Comité contre la torture, Doc off AG NU, 69 ${ }^{\mathrm{e}}$ sess, supp 44, Doc NU A/69/44, annexe XIV. 
diligence signifie que l'État doit ouvrir une enquête sur-le-champ ou sans retard dès qu'il suspecte qu'il y a eu crime, l'exigence d'impartialité signifie en revanche que l'État doit procéder avec sérieux, efficacité et objectivité. Dans certains cas d'allégations de torture, le Comité contre la torture a recommandé que les enquêtes aient lieu «sous la surveillance de juges indépendants appartenant au pouvoir judiciaire ${ }^{169} »$. Dans d'autres cas, il a recommandé «[d]'interdire [formellement] à tout organisme d'État qui n'est pas habilité judiciairement pour ce faire de mener des enquêtes [en matière] pénale ${ }^{170} »$. Selon le Comité, une enquête impartiale suppose que l'on accorde le même poids aux déclarations à charge et à décharge et que l'on mette en œuvre tous les moyens d'investigation utiles, s'agissant par exemple de vérifier les dossiers officiels disponibles, d'entendre les fonctionnaires incriminés ou d'ordonner l'exhumation de corps ${ }^{171}$. Dans la plupart des cas où le Comité a conclu à un défaut de diligence, il n'avait été procédé à aucune enquête ou l'enquête était intervenue bien tardivement ${ }^{172}$.

D'après le commentaire, l'État territorial est le mieux placé pour mener pareille enquête, afin de déterminer si effectivement des crimes ont été commis ou sont en voie de l'être et, dans l'affirmative, s'ils sont le fait de forces gouvernementales sous son contrôle, de forces sous le contrôle d'un autre État ou de membres d'une organisation non étatique ${ }^{173}$.

Si cette enquête immédiate et impartiale doit être menée par l'État sur le territoire duquel il y a "des motifs raisonnables de croire » que les crimes contre l'humanité ont été commis ou sont en voie d'être commis, indépendamment de la question de savoir si les auteurs sont préalablement identifiés sur le territoire d'un État ou que les victimes ont officiellement porté plainte auprès des autorités de ce dernier, elle est à distinguer de l'enquête préliminaire que doit mener l'État qui a la garde du suspect. À cet effet, le projet d'article 8 impose à l'État sur le territoire sous la juridiction duquel l'auteur présumé de l'infraction se trouve de prendre certaines mesures préliminaires consistant d'une part, au placement de l'intéressé en détention ou prendre toutes autres mesures juridiques conformes à sa législation qui sont nécessaires pour assurer sa présence, ces mesures ne pouvant toutefois être maintenues que pendant le délai nécessaire à l'engagement de poursuites pénales ou d'une procédure d'extradition ou de remise. D'autre part, l'État est tenu de procéder immédiatement à une enquête préliminaire sur les faits. Cette dernière est destinée cette fois à établir les faits concernant tel ou tel auteur présumé bien identifié qui se trouve dans un État, laquelle est visée au paragraphe 2 du projet d'article 8 d'après lequel l'État est tenu de procéder immédiatement à une enquête préliminaire sur les

169 Ibid, chap IV, « Examen des rapports présentés par les États parties en application de l'article 19 de la Convention, Équateur » au para 105.

170 Ibid, chap IV, « Examen des rapports présentés par les États parties en application de l'article 19 de la Convention, Guatemala » au para $76 \mathrm{~d}$ ).

171 Voir Ibid, annexe VIII, sect A; Ben M'Barek c Tunisie, communication nº 60/1996, 10 novembre 1999 aux paras 11.9-11.10.

172 Projets d'articles sur les crimes contre l'humanité, supra note 11, commentaire 3 du projet d'article 7 à la p 280.

173 Ibid, Commentaire $1 \mathrm{du}$ projet d'article 7 à la p 279. 
faits ${ }^{174}$, l'objectif étant de déterminer si une infraction pouvant entraîner l'exercice de l'action publique a été commise ${ }^{175}$.

L'obligation de l'État de procéder à une enquête préliminaire instituée par le paragraphe $2 \mathrm{du}$ projet d'article 8 sur les crimes contre l'humanité trouve son équivalent dans l'article 6 de la Convention contre la torture ${ }^{176}$ sur le modèle duquel elle est calquée. D'autres conventions internationales relatives à la criminalité contiennent généralement des dispositions qui prévoient l'adoption de ce type de mesures préliminaires ${ }^{177}$. Ayant examiné, notamment, le contenu de l'article 6 de la Convention contre la torture, la Cour internationale de Justice a expliqué que

[1]'introduction en droit interne de la législation appropriée [...] permettrait à l'État sur le territoire duquel se trouve le suspect de procéder immédiatement à une enquête préliminaire en vue d'établir les faits [...], étape nécessaire pour que cet État puisse, en connaissance de cause, soumettre l'affaire à ses autorités compétentes pour l'exercice de l'action pénale $[\ldots]^{178}$.

La Cour a estimé que l'enquête préliminaire était destinée, comme toute enquête menée par les autorités compétentes, à corroborer ou non les soupçons qui pesaient sur l'intéressé. Qu'il revenait à ces autorités d'établir un dossier en rassemblant les faits et les éléments de preuve, "qu'il s'agisse de documents ou de témoignages se rapportant aux événements en cause et à l'implication éventuelle du suspect dans le contexte en question ${ }^{179} »$. La Cour a en outre fait observer que «le choix des moyens, pour mener l'enquête, rest[ait] entre les mains des États parties ", mais que «des mesures [devaient être] prises aussitôt que le suspect [était] identifié sur le territoire de l'État, afin de conduire une enquête au sujet de ladite affaire ${ }^{180} »$. De plus, l'objet de telles mesures préliminaires était de «permettre l'engagement de poursuites contre le suspect, à défaut d'extradition, et la réalisation de l'objet et du but de la Convention, qui est d'accroître l'efficacité de la lutte contre la torture, en évitant l'impunité des auteurs de tels actes ${ }^{181} »$.

Ibid, art 8.

175 Voir Ibid, commentaire 2) du projet d'article 8 à la p 281.

176 Convention contre la torture, supra note 59, art 6.

177 Voir par exemple, Convention sur la capture illicite d'aéronefs, art 6; Convention pour la répression d'actes illicites dirigés contre la sécurité de l'aviation civile, art 6; Convention internationale contre la prise d'otages, art 6; Convention interaméricaine pour la prévention et la répression de la torture, art 8; Convention internationale pour la répression des attentats terroristes à l'explosif, art 7; Convention internationale pour la répression du financement du terrorisme, art 9; Convention de l'Organisation de l'unité africaine sur la prévention et la lutte contre le terrorisme, art 7; Convention internationale pour la protection de toutes les personnes contre les disparitions forcées, art 10; Convention de l'Association des nations de l'Asie du Sud-Est sur la lutte contre le terrorisme, art VIII.

178 Belgique c Sénégal, supra note 111 au para 72.

179 Ibid au para 83.

180 Ibid au para 86.

181 Ibid au para 74. 
Le texte des projets d'articles sur les crimes contre l'humanité provisoirement adoptés par la CDI à sa session de 2016 confirme que les crimes contre l'humanité sont des crimes de droit international que les États s'engagent, de manière générale, à prévenir et à punir. Plus spécialement, l'État est tenu de prendre des mesures positives pour prévenir des crimes contre l'humanité sur tout territoire sous sa juridiction ou son contrôle, ces termes ayant été entendus comme référant à tout territoire sur lequel l'État possède une compétence légale, c'est-à-dire le titre légal à agir conféré par une norme de droit international coutumier ou conventionnel, ou tout territoire sur lequel l'État exerce une sorte de pouvoir de fait sans aucune compétence légale. Bien plus, l'État sur le territoire sous la juridiction duquel se trouve l'auteur présumé est obligé de poursuivre à défaut d'extrader ou de remise. Ceci revient à dire qu'il doit soumettre l'affaire à ses autorités compétentes pour l'exercice de l'action pénale, indépendamment d'une demande d'extradition vers un autre État ou de remise du présumé auteur à une juridiction internationale. Les autorités compétentes, qui d'après les commentaires de la CDI sont représentées par l'Officier du Ministère Public, se comportent de la même manière qu'elles le feraient s'agissant d'une infraction de droit commun de caractère grave. Elles peuvent, par conséquent, exercer sur l'affaire leur pouvoir d'appréciation de l'opportunité des poursuites. Ce qui peut conduire au classement sans suite du dossier, sans que cette décision contrevienne à l'obligation imposée à l'État dont ces autorités relèvent, de poursuivre le suspect à défaut de l'extrader ou de le remettre à une juridiction internationale compétente. Pratiquement, ceci amène toutefois à penser qu'il n'existe à charge des États ni obligation de juger les présumés auteurs, ni celle de les punir. L'obligation de soumettre l'affaire au Ministère Public pour l'exercice de l'action pénale ne veut pas dire non plus une obligation d'engager des poursuites. À cet égard, l'on a tendance à séparer les autorités compétentes chargées des poursuites de l'État lui-même. Certainement que c'est par souci de commodité avec les principes de séparation des pouvoirs et de l'indépendance de la justice. Mais, l'État étant une entité abstraite, elle ne peut agir qu'à travers les organes exerçant des prérogatives de la puissance publique en l'occurrence, les organes judiciaires. Par ailleurs, l'on sait que la nature particulière des crimes contre l'humanité fait qu'ils sont généralement commis par ou au nom de ceux qui exercent l'autorité de l'État. Dans ces circonstances, les autorités de l'État au nom desquelles les crimes ont été commis n'auront donc qu'un intérêt limité à poursuivre les auteurs desdits crimes. L'obligation de n'initier que la procédure en saisissant le Ministère Public pour l'exercice de l'action pénale telle que proposée par le texte des projets d'articles doit donc, à tout le moins, s'accompagner d'une obligation de développer un pouvoir judiciaire indépendant et efficace, qui contre toute influence de la machine étatique sur l'affaire, devra s'abstenir d'exercer un pouvoir illimité d'appréciation de l'opportunité des poursuites en ce qui concerne des crimes dont la nature est telle qu'ils portent gravement atteinte à la dignité humaine en tant que telle et qu'il s'agit là d'une valeur dont la protection transcende l'intérêt individuel des États et au respect de laquelle ceux-ci ont un intérêt commun et par ailleurs égal. Tout ceci interdit en tout cas de soutenir l'exercice d'un tel pouvoir pour des motifs autres que l'insuffisance des charges, à l'instar du défaut d'intérêt national dans les poursuites. 